\title{
Oligosaccharide Synthesis in Microreactors
}

Frédéric R. Carrel, Karolin Geyer, Jeroen D.C. Codée, and Peter H. Seeberger

Laboratory of Organic Chemistry, Swiss Federal Institute of Technology (ETH) Zürich, Wolfgang-Pauli-Strasse 10, 8093 Zürich, Switzerland E-mail: seeberger@org.chem.ethz.ch

\section{Supporting Information}

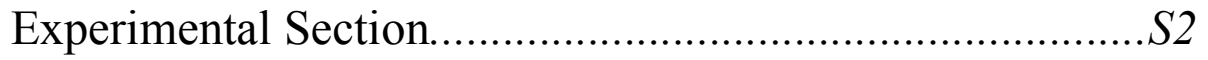

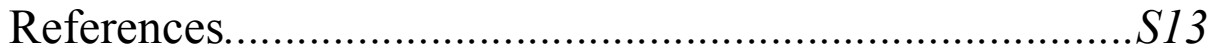

1H, 13C, 19F, 31P - NMR Spectra...................................S14 


\section{Experimental Section}

General methods. All chemicals were reagent grade and were used as supplied unless otherwise noted. All reactions were performed in oven-dried glassware under an inert atmosphere (nitrogen or argon) unless noted otherwise. Dichloromethane $\left(\mathrm{CH}_{2} \mathrm{Cl}_{2}\right)$ and tetrahydrofuran (THF) were purified by a J. C. Meyer Solvent Dispensing System (two packed columns of neutral alumina). Solvents for chromatography and work-up procedures were distilled from commercially available technical grade solvents. Analytical thin-layer chromatography was performed on E. Merck silica gel $60 \mathrm{~F}_{254}$ plates $(0.25 \mathrm{~mm})$. Compounds were visualized by UV and/or by dipping the plates in a cerium sulphate-ammonium molybdate solution followed by heating. Liquid column chromatography was performed using forced flow of the indicated solvent on Fluka silica gel $60(40-63 \mu \mathrm{m}) .{ }^{1} \mathrm{H}-,{ }^{13} \mathrm{C}-,{ }^{19} \mathrm{~F}-$, and ${ }^{31} \mathrm{P}-\mathrm{NMR}$ spectra were recorded on a Varian Mercury XL 300 spectrometer. The ${ }^{1} \mathrm{H}-\mathrm{NMR}$ spectra $(300 \mathrm{MHz})$ are expressed in $\mathrm{ppm}$ relative to $\mathrm{CHCl}_{3}(7.26 \mathrm{ppm})$ as internal reference, the coupling constants are reported in $\mathrm{Hz}$. The same is valid for ${ }^{13} \mathrm{C}-\mathrm{NMR}$ spectra $(75 \mathrm{MHz}$, internal reference $\left.\mathrm{CDCl}_{3}: 77.0 \mathrm{ppm}\right)$. For ${ }^{19} \mathrm{~F}-\mathrm{NMR}(282 \mathrm{MHz})$ and ${ }^{31} \mathrm{P}-\mathrm{NMR}$ spectra (121 $\mathrm{MHz})$ spectra, $\mathrm{CCl}_{3} \mathrm{~F}(\delta=0 \mathrm{ppm})$ and $\mathrm{H}_{3} \mathrm{PO}_{4}(\delta=0 \mathrm{ppm})$ were used as internal references. Optical rotations $[\alpha]_{\mathrm{D}}$ were recorded on a Jasco DIP-370 spectrometer using a sodium lamp $(\lambda=589 \mathrm{~nm})$ at room temperature with a $10 \mathrm{~cm} / 1 \mathrm{~mL}$ cell. The solvent and the concentration are specified, e.g. $c=1=10 \mathrm{mg} / \mathrm{mL}$. IR spectra were recorded in $\mathrm{CHCl}_{3}$ on a Perkin-Elmer 1600 FT-IR spectrometer and are expressed in $\mathrm{cm}^{-1}$. LC MS spectra were obtained using a Agilent 1100 LC MSD high-performance liquid chromatograph with a Waters Symmetry ${ }^{\circledR}$ C18 column $(3.9 \times 150 \mathrm{~nm}, 5 \mu \mathrm{m})$, and a gradient of acetonitrile/iso-propanol (80/20) and water/iso-propanol (80/20) as the mobile phase (flow rate $1 \mathrm{~mL} \mathrm{~min}^{-1}$; if necessary, $0.1 \%$ TFA was added to the eluents). The spectra were detected at 208, 234, 254 and $280 \mathrm{~nm}$. Preparative HPLC was performed using a Waters HPLC apparatus with a Waters SunFire ${ }^{\mathrm{TM}}$ Prep C8 column $(10 \times 150 \mathrm{~mm}, 5 \mu \mathrm{m})$, and a gradient of acetonitrile/iso-propanol (80/20, 0.1\% TFA) and water/iso-propanol $(80 / 20,0.1 \% \mathrm{TFA})$ as the mobile phase (flow rate $\left.5 \mathrm{~mL} \cdot \mathrm{min}^{-1}\right)$. The spectra were detected at a wavelength of 208 and $254 \mathrm{~nm}$. High-resolution mass spectroscopy (HRMS) was performed by the MS-service at the Laboratory of Organic Chemistry at ETH Zürich; 2,5-dihydroxybenzoic acid (DHB) was used as matrix. 
Dibutyl (3,4-di-O-benzyl-6-O-[fluorenylmethoxycarbonyl]-2- $O$-pivaloyl- $\beta$-Dglucopyranosyl) phosphate (1) was synthesized according to a published procedure. ${ }^{1}$

$8,8,9,9,10,10,11,11,12,12,13,13,14,14,15,15,15$-Heptadecafluoropentadec-4(Z)-en1-ol (2): To a solution of $7(647 \mathrm{mg}, 0.835 \mathrm{mmol})$ in THF $(12.5 \mathrm{~mL})$ was added $\mathrm{HCl}(1 \mathrm{M}$ in $\mathrm{H}_{2} \mathrm{O}, 4.2 \mathrm{~mL}, 4.2 \mathrm{mmol}$ ) at room temperature. The solution was refluxed for $20 \mathrm{~h}$, and then allowed to cool to room temperature. The reaction mixture was diluted with EtOAc $(100 \mathrm{~mL})$ and treated with a saturated aqueous $\mathrm{NaHCO}_{3}$ solution $(40 \mathrm{~mL})$. The aqueous phase was extracted with EtOAc $(2 \times 50 \mathrm{~mL})$, and the combined organic phases were dried over $\mathrm{MgSO}_{4}$ and concentrated. The residue was purified by silica gel flash column chromatography (EtOAc/cyclohexane, gradient 1:9 to 1:2) to afford $2\left(352 \mathrm{mg}, 79 \%\right.$ ) as a white solid. $\mathrm{R}_{\mathrm{f}} 0.25$ $($ EtOAc/cyclohexane $=1: 4) ;{ }^{1} \mathrm{H}-\mathrm{NMR}\left(300 \mathrm{MHz}, \mathrm{CDCl}_{3}\right) \delta 5.49$ (ddddd, $J=10.9,7.1,7.1$, $1.3,1.3 \mathrm{~Hz}, 1 \mathrm{H}), 5.37$ (ddd, $J=10.9,7.1,7.1 \mathrm{~Hz}, 1 \mathrm{H}), 3.66(\mathrm{t}, J=6.5 \mathrm{~Hz}, 2 \mathrm{H}), 2.42-2.31$ (m, $\left.2 \mathrm{H}, \mathrm{CH}_{2}\right), 2.21-2.01(\mathrm{~m}, 4 \mathrm{H}), 1.64$ (quint., $\left.J=6.5 \mathrm{~Hz}, 2 \mathrm{H}\right), 1.40(\mathrm{bs}, 1 \mathrm{H}) ;{ }^{13} \mathrm{C}-\mathrm{NMR}(75$ $\left.\mathrm{MHz}, \mathrm{CDCl}_{3}\right) \delta 131.2,126.8,62.2,30.8(\mathrm{t}, J=22 \mathrm{~Hz}), 32.2,23.3,18.1 ;{ }^{19} \mathrm{~F}-\mathrm{NMR}(282 \mathrm{MHz}$, $\left.\mathrm{CDCl}_{3}\right) \delta-80.7(\mathrm{t}, J=9.6 \mathrm{~Hz}, 3 \mathrm{~F}),-114.4--114.7$ (quint., $\left.2 \mathrm{~F}\right),-121.4--122.1(\mathrm{~m}, 6 \mathrm{~F}),-122.7$ (bs, 2F), -123.5 (bs, 2F), -126.1 (bs, 2F); IR $\left(\mathrm{CHCl}_{3}\right)$ : 3618, 3443, 3008, 2939, 1448, 1200, 1152, 1113, 1060, 973, $876 \mathrm{~cm}^{-1}$; HRMS-ESI Calcd for $\mathrm{C}_{15} \mathrm{H}_{11} \mathrm{~F}_{17} 514.0584\left(\mathrm{M}-\mathrm{H}_{2} \mathrm{O}\right)^{+}$; Found: 514.0581.

4-Trityloxybutan-1-ol (3). To a vigorously stirred solution of 1,4-butanediol (27.3 g, $300 \mathrm{mmol})$ in $\mathrm{CH}_{2} \mathrm{Cl}_{2}(300 \mathrm{~mL})$ were added $\mathrm{TrCl}(8.363 \mathrm{~g}, 30 \mathrm{mmol})$ and pyridine $(4.83 \mathrm{~mL}$, $60 \mathrm{mmol}$ ) at room temperature. After $90 \mathrm{~min}$, the solution was washed with brine ( $3 \times 50 \mathrm{~mL}$ ). The organic phase was dried over $\mathrm{MgSO}_{4}$ and the solvents were evaporated in vacuo. The residue was purified by silica gel flash column chromatography $($ EtOAc/cyclohexane $=1: 2)$ to afford $3(7.90 \mathrm{~g}, 79 \%)$ as a white solid. Characterization data were consistent with the previously reported data. ${ }^{2}$

1-Iodo-4-trityloxybutane (4). To a solution of 3 (7.9 g, $23.7 \mathrm{mmol})$ in $\mathrm{Et}_{2} \mathrm{O}(90 \mathrm{~mL})$ and $\mathrm{CH}_{3} \mathrm{CN}(30 \mathrm{~mL})$ were added imidazole $(4.85 \mathrm{~g}, 71.3 \mathrm{mmol})$ and triphenylphosphine (9.35 $\mathrm{g}, 35.6 \mathrm{mmol})$ at room temperature. The solution was cooled to $0{ }^{\circ} \mathrm{C}$ and iodine $(9.05 \mathrm{~g}, 35.6$ mmol) was added in portions over $5 \mathrm{~min}$. After $30 \mathrm{~min}$ at $0{ }^{\circ} \mathrm{C}$, the solution was allowed to warm to room temperature overnight. After $16 \mathrm{~h}$ at room temperature, the solution was diluted with $\mathrm{Et}_{2} \mathrm{O}(300 \mathrm{~mL})$. The precipitate was filtered off and washed with $\mathrm{Et}_{2} \mathrm{O}(2 \times 50 \mathrm{~mL})$. The 
filtrate was washed with a saturated aqueous $\mathrm{Na}_{2} \mathrm{~S}_{2} \mathrm{O}_{3}$ solution $(2 \times 50 \mathrm{~mL})$, and dried over $\mathrm{MgSO}_{4}$. The solvents were evaporated in vacuo and the residue was purified by silica gel flash column chromatography (EtOAc/cyclohexane $=1: 19)$ to afford $4(8.66 \mathrm{~g}, 82 \%)$ as a white solid. Characterization data were consistent with the previously reported data. ${ }^{2}$

(4-Trityloxybutan)-triphenylphosphonium iodide (5). To a solution of 4 (8.66 g, $19.58 \mathrm{mmol})$ in benzene $(80 \mathrm{~mL})$ was added triphenylphosphine $(10.27 \mathrm{~g}, 39.16 \mathrm{mmol})$ at room temperature. The solution was heated under reflux for $17 \mathrm{~h}$, and then allowed to cool to room temperature. The phosphonium salt was filtered off, washed with benzene $(3 \times 30 \mathrm{~mL})$, and dried under vacuum to afford 5 (9.392 g, 68\%) as a white solid. ${ }^{1} \mathrm{H}-\mathrm{NMR}(300 \mathrm{MHz}$, $\left.\mathrm{CDCl}_{3}\right) \delta 7.80-7.60(\mathrm{~m}, 15 \mathrm{H}), 7.30-7.16(\mathrm{~m}, 15 \mathrm{H}), 3.60-3.46(\mathrm{~m}, 2 \mathrm{H}), 3.12(\mathrm{t}, J=5.6 \mathrm{~Hz}$, 2H), 2.00 (quint., $J=5.9 \mathrm{~Hz}, 2 \mathrm{H}), 1.86-1.74(\mathrm{~m}, 2 \mathrm{H}) ;{ }^{13} \mathrm{C}-\mathrm{NMR}\left(75 \mathrm{MHz}, \mathrm{CDCl}_{3}\right) \delta 143.7$, 135.0, 133.5 (d, $J=10 \mathrm{~Hz}), 130.4(\mathrm{~d}, J=10 \mathrm{~Hz}), 128.4,127.7,126.9,117.9$ (d, $J=85 \mathrm{~Hz})$, 86.5, 61.8, $29.8(\mathrm{~d}, J=16 \mathrm{~Hz}), 22.4(\mathrm{~d}, J=51 \mathrm{~Hz}), 19.7 ;{ }^{31} \mathrm{P}-\mathrm{NMR}\left(121 \mathrm{MHz}, \mathrm{CDCl}_{3}\right) \delta 24.3$; IR $\left(\mathrm{CHCl}_{3}\right):$ 3391, 3087, 3060, 3007, 2942, 2876, 1969, 1818, 1589, 1488, 1439, 1390, 1316, 1200, 1152, 1113, 1072, 1034, 998, $900 \mathrm{~cm}^{-1}$; HRMS-MALDI Calcd for $\mathrm{C}_{41} \mathrm{H}_{38} \mathrm{OP} 577.2655$ $(\mathrm{M}-\mathrm{I})^{+}$; Found: 577.2648 .

4,4,5,5,6,6,7,7,8,8,9,9,10,10,11,11,11-Heptadecafluoro-undecan-1-al (6). To a solution of 3-(perfluorooctyl)propan-1-ol (4.78 g, $10 \mathrm{mmol})$ in $\mathrm{CH}_{2} \mathrm{Cl}_{2}(200 \mathrm{~mL})$ was added Dess-Martin periodinane $(4.24 \mathrm{~g}, 10 \mathrm{mmol})$ at room temperature. After $30 \mathrm{~min}$, further DessMartin periodinane $(2.12 \mathrm{~g}, 5 \mathrm{mmol})$ was added and the solution was stirred for another 30 min. The solution was poured into a saturated aqueous $\mathrm{NaHCO}_{3} / \mathrm{Na}_{2} \mathrm{~S}_{2} \mathrm{O}_{3}$ solution $(150 \mathrm{~mL})$ and stirred for $30 \mathrm{~min}$. The aqueous phase was extracted with $\mathrm{CH}_{2} \mathrm{Cl}_{2}(200 \mathrm{~mL})$. The combined organic phases were dried over $\mathrm{MgSO}_{4}$ and silica gel $(20 \mathrm{~g})$ was added. The solvents were evaporated in vacuo and the adsorbed residue was purified by silica gel flash column chromatography (EtOAc/cyclohexane $=1: 2)$ to afford $6(3.502 \mathrm{~g}, 73 \%)$ as a colorless liquid. Characterization data were consistent with the previously reported data. ${ }^{3}$

\section{1,1,1,2,2,3,3,4,4,5,5,6,6,7,7,8,8-Heptadecafluoro-15-trityloxy-pentadec-11(Z)-ene (7).} To a solution of $3(7.47 \mathrm{~g}, 10.6 \mathrm{mmol})$ in THF $(50 \mathrm{~mL})$ was added BuLi (1.6 M in hexane, 6.6 $\mathrm{mL}, 10.6 \mathrm{mmol}$ ) at $-30^{\circ} \mathrm{C}$. The orange solution was stirred for $15 \mathrm{~min}$ at $-30{ }^{\circ} \mathrm{C}$ and a solution of 4 (2.52 g, $5.3 \mathrm{mmol})$ in THF $(10 \mathrm{~mL})$ was added. The resulting solution was stirred for 15 min at $-30{ }^{\circ} \mathrm{C}$ and then allowed to warm to room temperature for $30 \mathrm{~min}$. The solution was 
diluted with $\mathrm{CH}_{2} \mathrm{Cl}_{2}(200 \mathrm{~mL})$ and treated with a saturated aqueous $\mathrm{NaHCO}_{3}$ solution (50 $\mathrm{mL})$. The aqueous phase was extracted with $\mathrm{CH}_{2} \mathrm{Cl}_{2}(2 \times 100 \mathrm{~mL})$. The combined organic phases were dried over $\mathrm{MgSO}_{4}$ and the solvents were evaporated in vacuo. The residue was purified by silica gel flash column chromatography $\left(\mathrm{CH}_{2} \mathrm{Cl}_{2}\right.$ pure) to afford $7(3.68 \mathrm{~g}, 90 \%)$ as a yellowish oil. $\mathrm{R}_{\mathrm{f}} 0.78($ EtOAc/cyclohexane $=1: 9) ;{ }^{1} \mathrm{H}-\mathrm{NMR}\left(300 \mathrm{MHz}, \mathrm{CDCl}_{3}\right) \delta$ 7.50-7.44 (m, 6H), 7.36-7.23 (m, 9H), 5.46 (ddddd, $J=11.0,7.1,7.1,1.3,1.3 \mathrm{~Hz}, 1 \mathrm{H}), 5.33$ (ddd, 1H, $J=11.0,7.1,7.1 \mathrm{~Hz}, 1 \mathrm{H}), 3.17(\mathrm{t}, J=6.6 \mathrm{~Hz}, 2 \mathrm{H}), 2.38-2.28(\mathrm{~m}, 2 \mathrm{H}), 2.23-1.98(\mathrm{~m}, 4 \mathrm{H})$, 1.77-1.66 (m, 2H); ${ }^{13} \mathrm{C}-\mathrm{NMR}\left(75 \mathrm{MHz}, \mathrm{CDCl}_{3}\right) \delta 144.3,131.6,128.6,127.6,126.8,126.4$, 86.3, 62.9, $30.8(\mathrm{t}, J=22 \mathrm{~Hz}), 29.8,23.9,18.1 ;{ }^{19} \mathrm{~F}-\mathrm{NMR}\left(282 \mathrm{MHz}, \mathrm{CDCl}_{3}\right) \delta-80.7$ (t, $J=9.9 \mathrm{~Hz}, 3 \mathrm{~F}$ ), -114.4- -114.6 (quint., 2F), -121.4- -122.0 (m, 6F), -122.6 (bs, 2F), -123.4 (bs, 2F), -126.0 (bs, 2F); IR $\left(\mathrm{CHCl}_{3}\right): 3032,2943,1601,1490,1448,1200,1152 \mathrm{~cm}^{-1}$; HRMS-ESI Calcd for $\mathrm{C}_{28} \mathrm{H}_{22} \mathrm{~F}_{17} \mathrm{O} 697.1594\left(\mathrm{M}-\mathrm{C}_{6} \mathrm{H}_{5}\right)^{+}$; Found: 697.1590.

\section{Optimization of Glycosylation Conditions Using the Microreactor: General Procedure.}

Prior to introduction of the reagents, the microreactor was rinsed with 20 - 50 reactor volumes of each anhydrous tetrahydrofuran and the anhydrous solvent used in the reaction. Reagent solutions were prepared by azeotroping the building blocks separately with toluene and drying under high-vacuum. The reagents were diluted with anhydrous solvent to the desired concentrations (Table 2). The gastight syringes were flushed with anhydrous solvents before loading the reagents. Inlet 1 was used for the nucleophile $(2.5 \mathrm{~mL}$ syringe), inlet 2 for the glycosylating agent 1 (2.5 mL syringe), inlet 3 for the activator TMSOTf (1.0 mL syringe) and inlet 4 for a mixture of piperidine/DMF (1:4), also containing methyl 2,3,4,6-tetra- $O$ benzyl- $\alpha$-D-glucopyranose $\left[\begin{array}{ll}0.06 & \mathrm{M}\end{array}\right]$ for LC MS analysis and TBAF. Inlet 3 was preconditioned with $1 \mathrm{~mL}$ activator solution. After loading, the syringes were connected to the device and the microreactor was set to the desired temperature by an ice bath. A pre-quenched volume of $100 \mu \mathrm{L}$ was discarded at the desired flow rate to flush the device, followed by the collection of 3 aliquots of $25 \mu \mathrm{L}$ of reaction mixture diluted with $1 \mathrm{~mL}$ acetonitrile for analysis. Seven flow rates were screened $\left(469.8 \mu \mathrm{L} \cdot \mathrm{min}^{-1}, 234.9 \mu \mathrm{L} \cdot \mathrm{min}^{-1}, 156.6 \mu \mathrm{L} \cdot \mathrm{min}^{-1}\right.$, $78.3 \mu \mathrm{L} \cdot \min ^{-1}, 39.15 \mu \mathrm{L} \cdot \min ^{-1}, 15.66 \mu \mathrm{L} \cdot \min ^{-1}$ and $\left.7.8 \mu \mathrm{L} \cdot \mathrm{min}^{-1}\right)$, corresponding to reaction times of $10 \mathrm{~s}, 20 \mathrm{~s}, 30 \mathrm{~s}, 60 \mathrm{~s}, 120 \mathrm{~s}, 300 \mathrm{~s}$ and $600 \mathrm{~s}$. The results depicted in Graphs 2-8 are summarized in Table 1. 
Table 1

\begin{tabular}{|c|c|c|c|c|c|c|c|c|}
\hline Entry & $\begin{array}{c}\text { Nucleophile } \\
(1 \text { equiv.) }\end{array}$ & $\begin{array}{c}\text { Building Block 1 } \\
\text { (equiv.); } 0.24 \mathrm{M}\end{array}$ & $\begin{array}{c}\text { TMSOTf } \\
\text { (equiv.); } 0.6 \mathrm{M}\end{array}$ & Solvent & $\begin{array}{c}\text { Temp } \\
{\left[{ }^{\circ} \mathrm{C}\right]}\end{array}$ & $\begin{array}{c}\text { TBAF } \\
\text { (equiv.); } 0.08 \mathrm{M}\end{array}$ & $\begin{array}{c}\text { Time } \\
{[\mathrm{s}]}\end{array}$ & Product \\
\hline 1 & $\mathbf{2} ; 0.12 \mathrm{M}$ & 2 & 2 & $\mathrm{TFT}$ & 0 & - & 60 & $\mathbf{8}^{*}$ \\
\hline 2 & $\mathbf{2} ; 0.12 \mathrm{M}$ & 2 & 2 & $\mathrm{TFT}$ & 20 & - & 30 & $\mathbf{8}^{*}$ \\
\hline 3 & $\mathbf{8} ; 0.12 \mathrm{M}$ & 2 & 2 & $\mathrm{CH}_{2} \mathrm{Cl}_{2}$ & 20 & - & 20 & $\mathbf{9}^{*}$ \\
\hline $4 \mathrm{a}$ & $\mathbf{9} ; 0.12 \mathrm{M}$ & 2 & 2 & $\mathrm{CH}_{2} \mathrm{Cl}_{2}$ & 20 & - & 30 & $\mathbf{1 0}^{*}$, residual 9 \\
\hline $4 \mathrm{~b}$ & $\mathbf{9} ; 0.12 \mathrm{M}$ & 2 & 2 & $\mathrm{CH}_{2} \mathrm{Cl}_{2}$ & 20 & $2^{* *}$ & 30 & $\mathbf{1 0}$, residual $\mathbf{9}$ \\
\hline 5 & $\mathbf{9} ; 0.08 \mathrm{M}$ & 3 & 3 & $\mathrm{CH}_{2} \mathrm{Cl}_{2}$ & 20 & 2 & 60 & $\mathbf{1 0}$ \\
\hline 6 & $\mathbf{1 0} ; 0.08 \mathrm{M}$ & 3 & 3 & $\mathrm{CH}_{2} \mathrm{Cl}_{2}$ & 20 & 2 & 60 & $\mathbf{1 1}$ \\
\hline
\end{tabular}

* Contaminated with the C(6)-TMS derivative. ** TBAF was added to the combined aliquots before FSPE

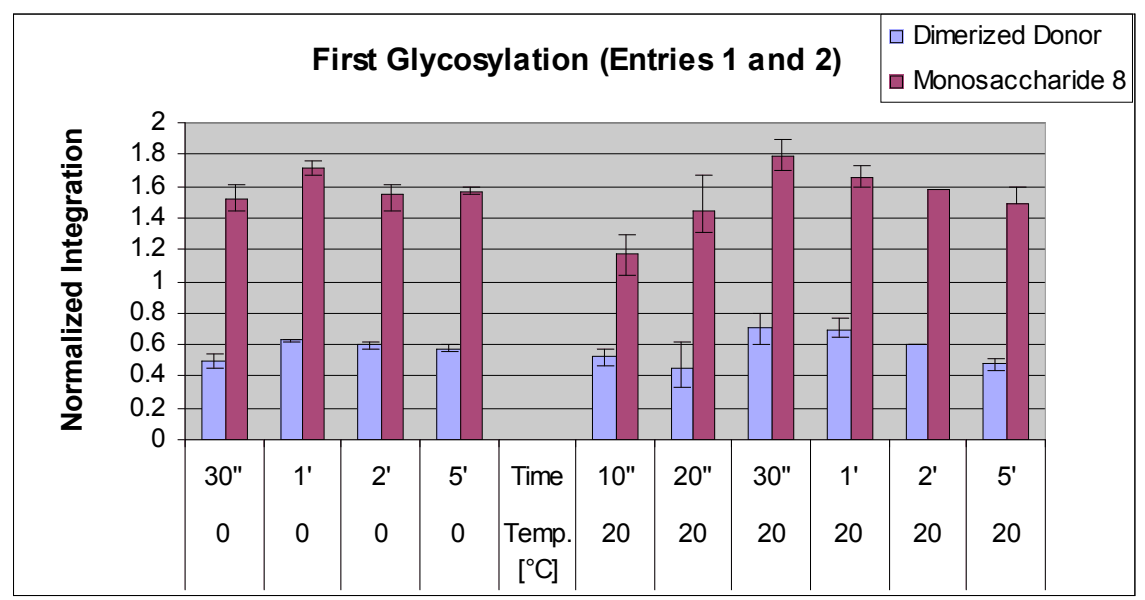

Graph 1: $1^{\text {st }}$ glycosylation at temperature of $0^{\circ} \mathrm{C}$ and $20{ }^{\circ} \mathrm{C}$

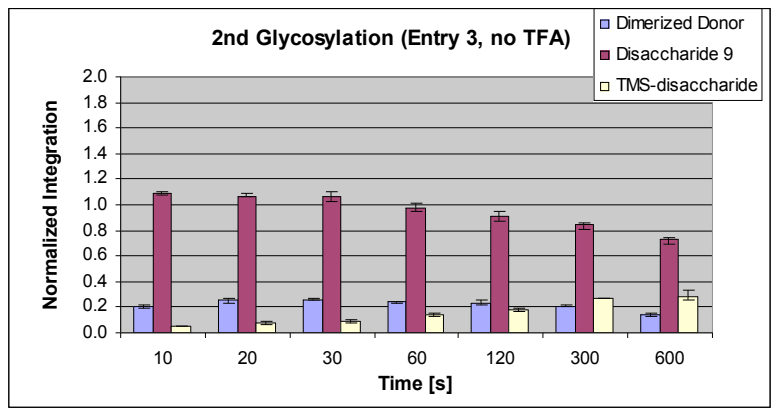

Graph2

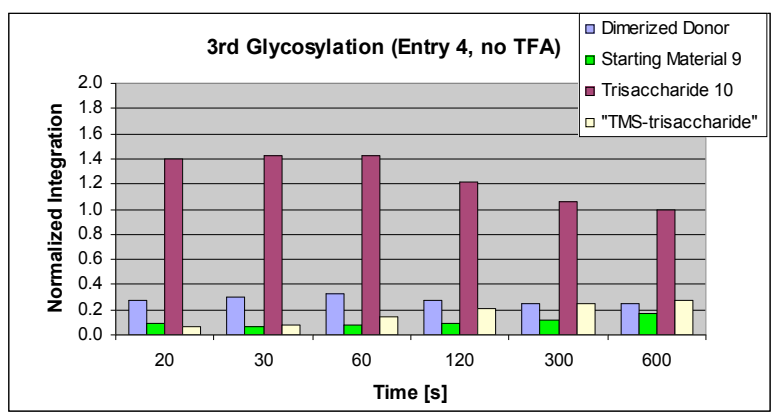

Graph4

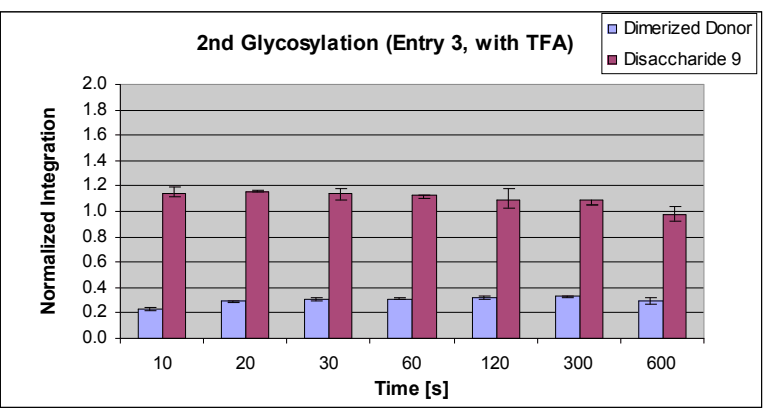

Graph3

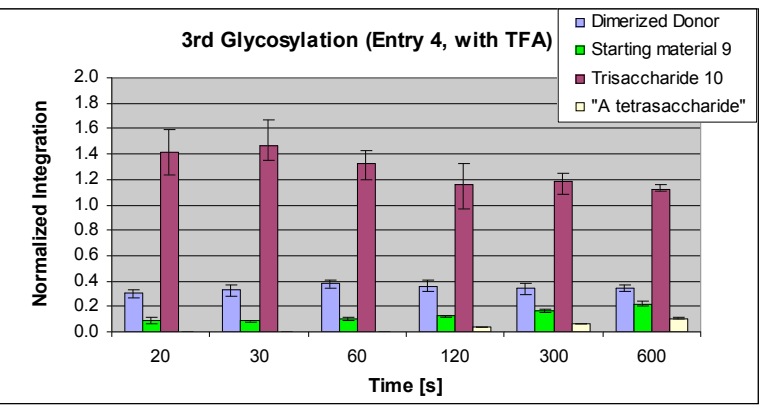

Graph5 


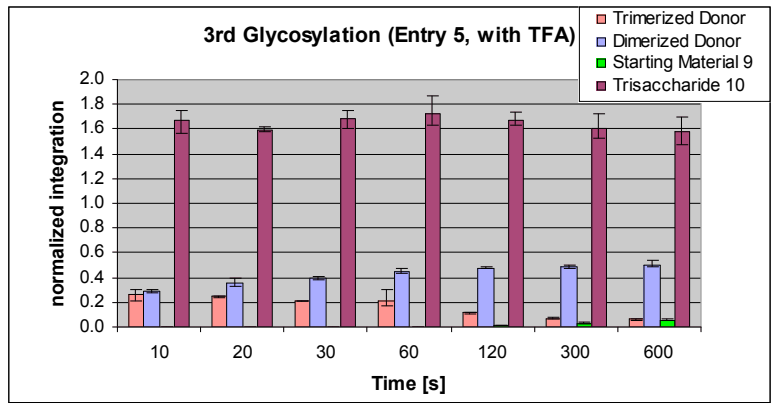

Graph6

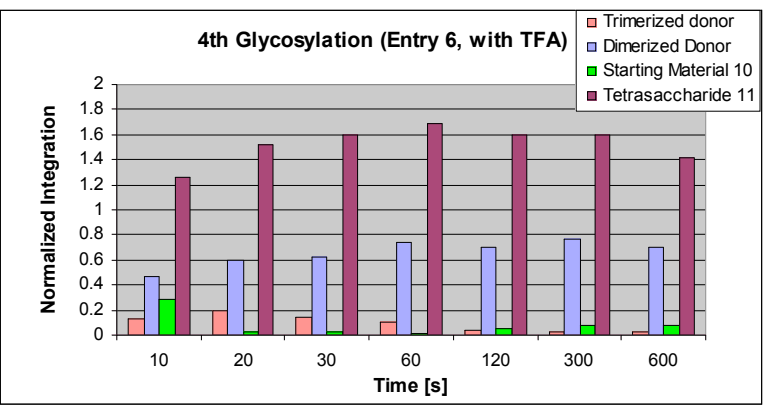

Graph 7

(only one data point, since small amounts)

\section{General Procedure for Scale-Out Using the Microreactor.}

Microreactor and gastight syringes were prepared according to the screening procedure. The building blocks were coevaporated with toluene and dried under high-vacuum overnight. Both reactants were dissolved in the solvent of the reaction to give solutions with defined concentrations (Table 2, optimized reaction conditions). TMSOTf was dissolved in the solvent of the reaction to the corresponding defined solution, TBAF was dissolved in a mixture of piperidine/DMF $=1: 4(\mathrm{v} / \mathrm{v})$. After flushing the microreactor and the syringes, the gastight glass syringes were filled with the corresponding reagent solutions and connected to the microreactor system. The reaction was carried out at the optimal reaction temperature and reaction time, whereby the first $300 \mu \mathrm{L}$ of pre-quenched volume were discarded, until the stock solutions were consumed. The solvents were evaporated in vacuo and residual DMF and piperidine were coevaporated with toluene $(3 \times 5 \mathrm{~mL})$. The residue was purified by FSPE ("Si-Tridecafluoro, Silicycle", loading with $\mathrm{DMF} / \mathrm{H}_{2} \mathrm{O}=9: 1$ ), using the eluent $\mathrm{MeOH} / \mathrm{H}_{2} \mathrm{O}=$ 8:2 for non-fluorinated fractions and $\mathrm{MeOH}$ for the fluorinated fractions. The methanol fractions were evaporated in vacuo and residual solvents were coevaporated with toluene/methanol in vacuo. The residue was dissolved in $\mathrm{CH}_{2} \mathrm{Cl}_{2}$ and dried over $\mathrm{MgSO}_{4}$. After evaporation of the solvents in vacuo, the corresponding glycoside was obtained as a white foam and was used for the next glycosylation.

Table 2

\begin{tabular}{|c|c|c|c|c|c|c|c|c|}
\hline Entry & $\begin{array}{c}\text { Nucleophile } \\
(1 \text { equiv.) }\end{array}$ & $\begin{array}{c}\text { Building Block 1 } \\
\text { (equiv.); } 0.24 \mathrm{M}\end{array}$ & $\begin{array}{c}\text { TMSOTf } \\
\text { (equiv.); } 0.6 \mathrm{M}\end{array}$ & Solvent & $\begin{array}{c}\text { TBAF } \\
\text { (equiv.) }\end{array}$ & $\begin{array}{c}\text { Temp } \\
{\left[{ }^{\circ} \mathrm{C}\right]}\end{array}$ & $\begin{array}{c}\text { time } \\
{[\mathrm{s}]}\end{array}$ & $\begin{array}{c}\text { Product } \\
\text { (yield) }\end{array}$ \\
\hline 1 & $\mathbf{2} ; 0.12 \mathrm{M}$ & 2 & 2 & $\mathrm{TFT}$ & $1.5 ; 0.09 \mathrm{M}$ & 20 & 30 & $\mathbf{8}(99 \%)$ \\
\hline 2 & $\mathbf{8} ; 0.12 \mathrm{M}$ & 2 & 2 & $\mathrm{CH}_{2} \mathrm{Cl}_{2}$ & $1.5 ; 0.09 \mathrm{M}$ & 20 & 20 & $\mathbf{9}(97 \%)$ \\
\hline 3 & $\mathbf{9} ; 0.08 \mathrm{M}$ & 3 & 3 & $\mathrm{CH}_{2} \mathrm{Cl}_{2}$ & $2 ; 0.08 \mathrm{M}$ & 20 & 60 & $\mathbf{1 0}(90 \%)$ \\
\hline 4 & $\mathbf{1 0} ; 0.08 \mathrm{M}$ & 3 & 3 & $\mathrm{CH}_{2} \mathrm{Cl}_{2}$ & $2 ; 0.08 \mathrm{M}$ & 20 & 60 & $\mathbf{1 1}(95 \%)$ \\
\hline
\end{tabular}


$[8,8,9,9,10,10,11,11,12,12,13,13,14,14,15,15,15-H e p t a d e c a f l u o r o p e n t a d e c-4(Z)$-en-

1-yl] 3,4-di- $\boldsymbol{O}$-benzyl-2- $\boldsymbol{O}$-pivaloyl- $\boldsymbol{\beta}$-D-glucopyranoside (8). General procedure using building blocks 2 (133 mg, $0.25 \mathrm{mmol}, 0.12 \mathrm{M}$ in TFT), 1 (430 mg, $0.50 \mathrm{mmol}, 0.24 \mathrm{M}$ in TFT), TMSOTf $(90 \mu \mathrm{L}, 0.50 \mathrm{mmol}, 0.6 \mathrm{M}$ in TFT) and TBAF (1M in THF, $0.375 \mathrm{mmol}$, $0.09 \mathrm{M}$ in piperidine/DMF $=1: 4)$ at $+20{ }^{\circ} \mathrm{C}$ and $30 \mathrm{~s}$ reaction time gave a total pre-quenched volume of $4.28 \mathrm{~mL}(0.214 \mathrm{mmol})$ which afforded $8(203 \mathrm{mg}, 99 \%)$ as a white foam. $\mathrm{R}_{\mathrm{f}} 0.63$ $($ EtOAc:cyclohexane $=1: 2) ;[\alpha]_{\mathrm{D}}{ }^{\mathrm{rt}}=-6.6^{\circ}\left(c=1, \mathrm{CHCl}_{3}\right) ;{ }^{1} \mathrm{H}-\mathrm{NMR}\left(300 \mathrm{MHz}, \mathrm{CDCl}_{3}\right) \delta$ $7.36-7.22(\mathrm{~m}, 10 \mathrm{H}), 5.50-5.31(\mathrm{~m}, 2 \mathrm{H}), 5.04(\mathrm{dd}, J=9.1,8.0 \mathrm{~Hz}, 1 \mathrm{H}), 4.82(\mathrm{~d}, J=11.0 \mathrm{~Hz}$, $1 \mathrm{H}), 4.78(\mathrm{~d}, J=11.0 \mathrm{~Hz}, 1 \mathrm{H}), 4.71(\mathrm{~d}, J=11.0 \mathrm{~Hz}, 1 \mathrm{H}), 4.63(\mathrm{~d}, J=11.0 \mathrm{~Hz}, 1 \mathrm{H}), 4.43$ (d, $J=8.0 \mathrm{~Hz}, 1 \mathrm{H}), 3.89$ (dd, $J=12.4,2.8 \mathrm{~Hz}, 1 \mathrm{H}), 3.85$ (ddd, $J=9.3,6.6,6.6 \mathrm{~Hz}, 1 \mathrm{H}), 3.74$ (dd, $J=9.0,9.0 \mathrm{~Hz}, 1 \mathrm{H}), 3.73(\mathrm{dd}, J=12.4,4.7 \mathrm{~Hz}, 1 \mathrm{H}), 3.67$ (dd, $J=9.0,9.0 \mathrm{~Hz}, 1 \mathrm{H}), 3.50-3.38$ (m, 2H), 2.40-2.29 (m, 2H), 2.21-2.01 (m, 4H), 1.90 (dd, $J=7.4,6.1 \mathrm{~Hz}, 1 \mathrm{H}), 1.64$ (quint., $J=6.8 \mathrm{~Hz}, 2 \mathrm{H}), 1.19(\mathrm{~s}, 9 \mathrm{H}) ;{ }^{13} \mathrm{C}-\mathrm{NMR}\left(75 \mathrm{MHz}, \mathrm{CDCl}_{3}\right) \delta 176.6,137.9,137.7,131.0$, 128.4, 128.3, 128.0, 127.3, 127.9, 127.6, 126.8, 101.2, 82.9, 77.7, 75.2, 75.0, 74.9, 72.9, 69.2, 61.8, 38.7, $30.8(\mathrm{t}, J=22 \mathrm{~Hz}), 29.3,27.0,23.5,18.1 ;{ }^{19} \mathrm{~F}-\mathrm{NMR}\left(282 \mathrm{MHz}, \mathrm{CDCl}_{3}\right) \delta-80.6(\mathrm{t}$, $J=9.6 \mathrm{~Hz}, 3 \mathrm{~F}),-114.3--114.6(\mathrm{~m}, 2 \mathrm{~F}),-121.4--122.0(\mathrm{~m}, 6 \mathrm{~F}),-122.6$ (bs, 2F), -123.4 (bs, 2F), -126.0 (m, 2F); IR ( $\left.\mathrm{CHCl}_{3}\right): 3600,3065,3008,2961,2874,1737,1602,1497,1479$, 1455, 1397, 1365, 1200, 1152, 1087, 1039, $866 \mathrm{~cm}^{-1}$; HRMS-MALDI Calcd for $\mathrm{C}_{40} \mathrm{H}_{43} \mathrm{~F}_{17} \mathrm{O}_{7} \mathrm{Na} 981.2630(\mathrm{M}+\mathrm{Na})^{+}$; Found: 981.2627.

\section{$[8,8,9,9,10,10,11,11,12,12,13,13,14,14,15,15,15$-Heptadecafluoropentadec-4(Z)-en-}

1-yl] 6-O-[3,4-di-O-benzyl-2-O-pivaloyl- $\beta$-D-glucopyranosyl]-3,4-di- $O$-benzyl-2- $O$ -

pivaloyl- $\beta$-D-glucopyranoside (9). General procedure using building blocks 8 (194 mg, $0.20 \mathrm{mmol}, 0.12 \mathrm{M}$ in $\left.\mathrm{CH}_{2} \mathrm{Cl}_{2}\right), 1$ (348 $\mathrm{mg}, 0.40 \mathrm{mmol}, 0.24 \mathrm{M}$ in $\left.\mathrm{CH}_{2} \mathrm{Cl}_{2}\right)$, TMSOTf $(73 \mu \mathrm{L}$, $0.40 \mathrm{mmol}, 0.6 \mathrm{M}$ in $\mathrm{CH}_{2} \mathrm{Cl}_{2}$ ) and TBAF (1M in THF, $0.30 \mathrm{mmol}, 0.09 \mathrm{M}$ in piperidine/DMF = $1: 4)$ at $+20{ }^{\circ} \mathrm{C}$ and $20 \mathrm{~s}$ reaction time gave a total pre-quenched volume of $3.53 \mathrm{~mL}(0.176$ mmol) which afforded $9(238 \mathrm{mg}, 97 \%)$ as a white foam. $\mathrm{R}_{\mathrm{f}} 0.67$ (EtOAc/cyclohexane = 1:2); $[\alpha]_{\mathrm{D}}{ }^{\mathrm{rt}}=-7.2^{\circ}\left(c=0.5, \mathrm{CHCl}_{3}\right) ;{ }^{1} \mathrm{H}-\mathrm{NMR}\left(300 \mathrm{MHz}, \mathrm{CDCl}_{3}\right) \delta 7.37-7.22(\mathrm{~m}, 20 \mathrm{H}), 5.51-5.30$ $(\mathrm{m}, J=10.7,7.1 \mathrm{~Hz}, 2 \mathrm{H}), 5.06(\mathrm{dd}, J=9.0,7.8 \mathrm{~Hz}, 1 \mathrm{H}), 5.02(\mathrm{dd}, J=9.3,8.1 \mathrm{~Hz}, 1 \mathrm{H}), 4.80$ (d, $J=11.2 \mathrm{~Hz}, 2 \mathrm{H}), 4.77$ (d, $J=11.2 \mathrm{~Hz}, 1 \mathrm{H}), 4.75(\mathrm{~d}, J=11.2 \mathrm{~Hz}, 1 \mathrm{H}), 4.69$ (d, $J=11.2 \mathrm{~Hz}, 1 \mathrm{H}), 4.69(\mathrm{~d}, J=11.2 \mathrm{~Hz}, 1 \mathrm{H}), 4.62(\mathrm{~d}, J=11.2 \mathrm{~Hz}, 1 \mathrm{H}), 4.59(\mathrm{~d}, J=11.2 \mathrm{~Hz}$, $1 \mathrm{H}), 4.51(\mathrm{~d}, J=7.8 \mathrm{~Hz}, 1 \mathrm{H}), 4.35(\mathrm{~d}, J=8.1 \mathrm{~Hz}, 1 \mathrm{H}), 3.95-3.36(\mathrm{~m}, 12 \mathrm{H}), 2.40-2.30(\mathrm{~m}$, 2H), 2.20-1.99 (m, 5H), 1.68-1.57 (quint., $J=7.2 \mathrm{~Hz}, 2 \mathrm{H}), 1.19$ (s, 9H), 1.18 (s, 9H); 
${ }^{13} \mathrm{C}-\mathrm{NMR}\left(75 \mathrm{MHz}, \mathrm{CDCl}_{3}\right) \delta$ 176.5, 137.9, 137.7, 137.6, 131.0, 128.4, 128.3, 128.0, 127.9, $127.8,127.5,127.3,127.2,126.8,101.1,100.9,83.0,77.9,75.6,75.3,75.0,74.9,74.8,72.9$, 69.1, 68.3, 61.9, 38.8, 30.9 (t, $J=22 \mathrm{~Hz}), 29.5,27.2,23.7,18.3 ;{ }^{19} \mathrm{~F}-\mathrm{NMR}\left(282 \mathrm{MHz}, \mathrm{CDCl}_{3}\right)$ $\delta$-80.6 (t, $J=9.6 \mathrm{~Hz}, 3 \mathrm{~F}),-114.0--114.3(\mathrm{~m}, 2 \mathrm{~F}),-121.1--121.7$ (m, 6F), -122.3 (bs, 2F), 123.0 (bs, 2F), -125.3 (m, 2F); IR ( $\left.\mathrm{CHCl}_{3}\right): 3600,3436,3008,2970,1737,1602,1524,1479$, 1454, 1397, 1361, 1200, 1146, 1090, 1042, 932, $874 \mathrm{~cm}^{-1}$; HRMS-MALDI Calcd for $\mathrm{C}_{65} \mathrm{H}_{73} \mathrm{~F}_{17} \mathrm{O}_{13} \mathrm{Na} 1407.4672(\mathrm{M}+\mathrm{Na})^{+}$; Found: 1407.4703 .

\section{$8,8,9,9,10,10,11,11,12,12,13,13,14,14,15,15,15-H e p t a d e c a f l u o r o p e n t a d e c-4(Z)$-en-}

\section{1-yl 6-O-\{6-O-[3,4-di- $O$-benzyl-2-O-pivaloyl- $\beta$-D-glucopyranosyl]-3,4-di- $O$-benzyl-2- $O$ -} pivaloyl- $\beta$-D-glucopyranosyl\}-3,4-di- $O$-benzyl-2- $O$-pivaloyl- $\beta$-D-glucopyranoside $\quad(10)$. General procedure using building blocks $9\left(221 \mathrm{mg}, 0.16 \mathrm{mmol}, 0.08 \mathrm{M}\right.$ in $\left.\mathrm{CH}_{2} \mathrm{Cl}_{2}\right), \mathbf{1}$ (411 mg, $0.479 \mathrm{mmol}, 0.24 \mathrm{M}$ in $\mathrm{CH}_{2} \mathrm{Cl}_{2}$ ), TMSOTf ( $86 \mu \mathrm{L}, 0.479 \mathrm{mmol}, 0.6 \mathrm{M}$ in $\mathrm{CH}_{2} \mathrm{Cl}_{2}$ ) and TBAF (1M in THF, $0.319 \mathrm{mmol}, 0.08 \mathrm{M}$ in piperidine/DMF $=1: 4$ ) at $+20{ }^{\circ} \mathrm{C}$ and $60 \mathrm{~s}$ reaction time gave a total pre-quenched volume of $3.06 \mathrm{~mL}(0.102 \mathrm{mmol})$ which afforded $10(166 \mathrm{mg}$, $90 \%)$ as a white foam. $\mathrm{R}_{\mathrm{f}}=0.74($ EtOAc/cyclohexane $=1: 2) ;[\alpha]_{\mathrm{D}}{ }^{\mathrm{rt}}=-7.8^{\circ}\left(c=0.5, \mathrm{CHCl}_{3}\right)$; ${ }^{1} \mathrm{H}-\mathrm{NMR}\left(300 \mathrm{MHz}, \mathrm{CDCl}_{3}\right) \delta 7.37-7.22(\mathrm{~m}, 30 \mathrm{H}), 5.47$ (ddd, $\left.J=10.7,7.2,7.2 \mathrm{~Hz}, 1 \mathrm{H}\right), 5.35$ (ddd, $J=10.7,7.2,7.2 \mathrm{~Hz}, 1 \mathrm{H}), 5.10-4.99$ (m, 3H), 4.81 (d, $J=11.0 \mathrm{~Hz}, 1 \mathrm{H}), 4.79$ (d, $J=11.0 \mathrm{~Hz}, 2 \mathrm{H}), 4.76(\mathrm{~d}, J=11.0 \mathrm{~Hz}, 1 \mathrm{H}), 4.75(\mathrm{~d}, J=11.0 \mathrm{~Hz}, 2 \mathrm{H}), 4.71(\mathrm{~d}, J=11.0 \mathrm{~Hz}$, $1 \mathrm{H}), 4.71(\mathrm{~d}, J=11.0 \mathrm{~Hz}, 1 \mathrm{H}), 4.68(\mathrm{~d}, J=11.0 \mathrm{~Hz}, 1 \mathrm{H}), 4.63(\mathrm{~d}, J=11.0 \mathrm{~Hz}, 1 \mathrm{H}), 4.61(\mathrm{~d}$, $J=11.0 \mathrm{~Hz}, 1 \mathrm{H}), 4.60(\mathrm{~d}, J=11.0 \mathrm{~Hz}, 1 \mathrm{H}), 4.50(\mathrm{~d}, J=7.7 \mathrm{~Hz}, 1 \mathrm{H}), 4.44(\mathrm{~d}, J=7.7 \mathrm{~Hz}, 1 \mathrm{H})$, $4.39(\mathrm{~d}, J=8.0 \mathrm{~Hz}, 1 \mathrm{H}), 3.98-3.33(\mathrm{~m}, 17 \mathrm{H}), 2.48(\mathrm{dd}, J=6.3,6.3 \mathrm{~Hz}, 1 \mathrm{H}), 2.39-2.29$ (m, 2H), 2.20-1.99 (m, 4H), 1.68-1.57 (quint., $J=7.0 \mathrm{~Hz}, 2 \mathrm{H}), 1.19$ (s, 9H), 1.19 (s, 9H), 1.17 (s, 9H) $;{ }^{13} \mathrm{C}-\mathrm{NMR}\left(75 \mathrm{MHz}, \mathrm{CDCl}_{3}\right) \delta 176.7,176.5,176.4,138.0,137.9,137.8,137.7,131.1$, $128.3,128.2$, 128.1, 127.9, 127.8, 127.5, 127.3, 127.2, 126.7, 101.9, 101.1, 100.7, 83.1, 82.9, 77.9, 77.6, 75.7, 75.0, 74.7, 73.0, 72.8, 69.3, 68.8, 68.3, 61.7, 38.7, 30.7 (t, $J=22 \mathrm{~Hz}), 29.4$, 27.0, 23.6, 18.2; ${ }^{19} \mathrm{~F}-\mathrm{NMR}\left(282 \mathrm{MHz}, \mathrm{CDCl}_{3}\right) \delta-80.6,-80.7(2 \mathrm{t}, J=9.6 \mathrm{~Hz}, 3 \mathrm{~F}),-114.3-$ 114.4, -115.9- -116.1 (2m, 2F), -121.3- -122.0 (m, 6F), -122.4- -123.0 (m, 2F), -123.4 (bs, 2F), -125.8--126.2 (m, 2F); IR $\left(\mathrm{CHCl}_{3}\right)$ : 3450, 3008, 2967, 2873, 1737, 1604, 1497, 1479, 454, 1398, 1363, 1200, 1144, $1088 \mathrm{~cm}^{-1}$; HRMS-MALDI Calcd for $\mathrm{C}_{90} \mathrm{H}_{103} \mathrm{~F}_{17} \mathrm{O}_{19} \mathrm{Na}$ $1833.6714(\mathrm{M}+\mathrm{Na})^{+}$, Found: 1833.6730 . 
$8,8,9,9,10,10,11,11,12,12,13,13,14,14,15,15,15-H e p t a d e c a f l u o r o p e n t a d e c-4(Z)$-en-

1-yl 6- $O$ - $\{6-O$-[6-O-(3,4-di- $O$-benzyl-2- $O$-pivaloyl- $\beta$-D-glucopyranosyl)-3,4-di- $O$-benzyl2-O-pivaloyl- $\beta$-D-glucopyranosyl]-3,4-di- $O$-benzyl-2- $O$-pivaloyl- $\beta$-D-glucopyranosyl $\}$ -

3,4-di- $\boldsymbol{O}$-benzyl-2- $\boldsymbol{O}$-pivaloyl- $\boldsymbol{\beta}$-D-glucopyranoside (11). General procedure using building blocks 10 (166 mg, $0.09 \mathrm{mmol}, 0.08 \mathrm{M}$ in $\left.\mathrm{CH}_{2} \mathrm{Cl}_{2}\right), 1(236 \mathrm{mg}, 0.275 \mathrm{mmol}, 0.24 \mathrm{M}$ in $\mathrm{CH}_{2} \mathrm{Cl}_{2}$ ), TMSOTf $\left(50 \mu \mathrm{L}, 0.275 \mathrm{mmol}, 0.6 \mathrm{M}\right.$ in $\left.\mathrm{CH}_{2} \mathrm{Cl}_{2}\right)$ and TBAF (1M in THF, $0.183 \mathrm{mmol}, 0.08 \mathrm{M}$ in piperidine $/ \mathrm{DMF}=1: 4)$ at $+20{ }^{\circ} \mathrm{C}$ and $60 \mathrm{~s}$ reaction time gave a total pre-quenched volume of $2.19 \mathrm{~mL}(0.073 \mathrm{mmol})$ which afforded $11(156 \mathrm{mg}, 95 \%)$ as a white foam. $\mathrm{R}_{\mathrm{f}} 0.75($ EtOAc/cyclohexane $=1: 2) ;[\alpha]_{\mathrm{D}}{ }^{\mathrm{rt}}=-8.0^{\circ}\left(c=0.5, \mathrm{CHCl}_{3}\right) ;{ }^{1} \mathrm{H}-\mathrm{NMR}(300$ $\left.\mathrm{MHz}, \mathrm{CDCl}_{3}\right) \delta 7.36-7.20(\mathrm{~m}, 40 \mathrm{H}), 5.47$ (ddd, $\left.J=10.7,7.1,7.1 \mathrm{~Hz}, 1 \mathrm{H}\right), 5.36$ (ddd, $J=10.7,7.1,7.1 \mathrm{~Hz}, 1 \mathrm{H}), 5.13-5.00(\mathrm{~m}, 4 \mathrm{H}), 4.84-4.58(\mathrm{~m}, 16 \mathrm{H}), 4.52(\mathrm{~d}, J=11.0 \mathrm{~Hz}, 1 \mathrm{H})$, $4.44(\mathrm{~d}, J=7.7 \mathrm{~Hz}, 1 \mathrm{H}), 4.43(\mathrm{~d}, J=7.7 \mathrm{~Hz}, 1 \mathrm{H}), 4.39$ (d, $J=8.2 \mathrm{~Hz}, 1 \mathrm{H}), 4.01$ (d, $J=10.0 \mathrm{~Hz}, 1 \mathrm{H}), 3.92-3.33(\mathrm{~m}, 21 \mathrm{H}), 2.66-2.55$ (bs, $1 \mathrm{H}), 2.40-2.30$ (m, 2H), 2.21-2.00 (m, 4H), 1.63 (quint., $J=6.9 \mathrm{~Hz}, 2 \mathrm{H}), 1.22(\mathrm{~s}, 9 \mathrm{H}), 1.20(\mathrm{~s}, 9 \mathrm{H}), 1.20(\mathrm{~s}, 9 \mathrm{H}), 1.19(\mathrm{~s}, 9 \mathrm{H}) ;{ }^{13} \mathrm{C}-$ NMR (75 MHz, $\left.\mathrm{CDCl}_{3}\right) \delta 176.8,176.6,176.5,176.4,138.0,137.9,137.9,137.7,131.1$, $128.4,128.3,128.2,127.9,127.9,127.8,127.7,127.4,127.2,127.2,126.7,102.0,101.6$, $100.7,83.1,82.9,78.3,77.7,77.2,75.7,75.0,74.9,74.7,74.3,73.1,72.9,72.5,69.4,69.0$, $68.7,67.8,61.7,38.7,30.7(\mathrm{t}, J=22 \mathrm{~Hz}), 29.4,27.1,27.0,27.0,23.6,18.1 ;{ }^{19} \mathrm{~F}-\mathrm{NMR}(282$ $\left.\mathrm{MHz}, \mathrm{CDCl}_{3}\right) \delta-80.6(\mathrm{t}, J=9.6 \mathrm{~Hz}, 3 \mathrm{~F}),-114.2--114.5$ (quint., $\left.2 \mathrm{~F}\right),-121.4--122.0$ (m, 6F), 122.5 (s, 2F), -123.3 (s, 2F), -125.8--126.1 (m, 2F); IR $\left(\mathrm{CHCl}_{3}\right)$ : 3066, 3008, 2967, 2935, 2874, 1737, 1602, 1497, 1479, 1454, 1397, 1363, 1277, 1200, 1144, $1085 \mathrm{~cm}^{-1}$; HRMSMALDI Calcd for $\mathrm{C}_{115} \mathrm{H}_{133} \mathrm{~F}_{17} \mathrm{O}_{25} \mathrm{Na} 2259.8757(\mathrm{M}+\mathrm{Na})^{+}$; Found: 2259.8818.

\section{$8,8,9,9,10,10,11,11,12,12,13,13,14,14,15,15,15-H e p t a d e c a f l u o r o p e n t a d e c-4(Z)$-en-} 1-yl 6-O-\{6-O-[6-O-(6-O-acetyl-3,4-di- $O$-benzyl-2- $O$-pivaloyl- $\beta$-D-glucopyranosyl)-3,4-di$O$-benzyl-2- $O$-pivaloyl- $\beta$-D-glucopyranosyl]-3,4-di- $O$-benzyl-2- $O$-pivaloyl- $\beta$-Dglucopyranosyl\}-3,4-di- $O$-benzyl-2- $O$-pivaloyl- $\beta$-D-glucopyranoside (12). To a solution of $11(29 \mathrm{mg}, 12.9 \mu \mathrm{mol})$ in pyridine $(0.5 \mathrm{~mL})$ was added acetic anhydride $(0.25 \mathrm{~mL})$ and DMAP (some crystals) at room temperature. After $4 \mathrm{~h}$, the solution was diluted with $\mathrm{CH}_{2} \mathrm{Cl}_{2}$ $(5 \mathrm{~mL})$ and treated with ice-water $(5 \mathrm{~mL})$. The aqueous phase was extracted with $\mathrm{CH}_{2} \mathrm{Cl}_{2}$ $(3 \times 5 \mathrm{~mL})$. The combined organic phases were washed with brine $(3 \mathrm{~mL})$ and dried over $\mathrm{MgSO}_{4}$. The solvents were evaporated in vacuo and the residue was purified by silica gel flash column chromatography (EtOAc/hexane =1:2) to afford $13(27 \mathrm{mg}, 92 \%)$ as a colorless 
oil. $\mathrm{R}_{\mathrm{f}} 0.72($ EtOAc/hexane $=1: 2) ;[\alpha]_{\mathrm{D}}{ }^{\mathrm{rt}}=-13.0^{\circ}\left(c=1.55, \mathrm{CHCl}_{3}\right) ;{ }^{1} \mathrm{H}-\mathrm{NMR}(300 \mathrm{MHz}$, $\left.\mathrm{CDCl}_{3}\right) \delta 7.38-7.16(\mathrm{~m}, 40 \mathrm{H}), 5.47(\mathrm{ddd}, J=10.6,7.0,7.0 \mathrm{~Hz}, 1 \mathrm{H}), 5.35(\mathrm{ddd}, J=10.6,6.7$, $6.7 \mathrm{~Hz}, 1 \mathrm{H}), 5.15-4.98(\mathrm{~m}, 4 \mathrm{H}), 4.79-4.55(\mathrm{~m}, 16 \mathrm{H}), 4.50-4.34(\mathrm{~m}, 4 \mathrm{H}), 4.29(\mathrm{dd}, J=11.2$, $1.6 \mathrm{~Hz}, 1 \mathrm{H}), 4.19(\mathrm{dd}, J=11.2,5.2 \mathrm{~Hz}, 1 \mathrm{H}), 4.06-3.34(\mathrm{~m}, 20 \mathrm{H}), 2.41-2.28(\mathrm{~m}, 2 \mathrm{H}), 2.21-$ $2.00(\mathrm{~m}, 4 \mathrm{H}), 1.99(\mathrm{~s}, 3 \mathrm{H}), 1.71-1.55(\mathrm{~m}, 2 \mathrm{H}), 1.19(\mathrm{~s}, 36 \mathrm{H}) ;{ }^{13} \mathrm{C}-\mathrm{NMR}\left(75 \mathrm{MHz}, \mathrm{CDCl}_{3}\right) \delta$ $176.7,176.4,170.5,138.1,137.8,131.5,131.2,128.4,128.3,128.2,128.0,127.9,127.8$, 127.6, 127.4, 127.2, 127.1, 127.1, 126.7, 101.4, 101.2, 100.8, 83.4, 83.1, 78.5, 78.3, 74.8, $72.9,72.7,68.7,63.2,38.7,32.0,29.6,27.1,27.0,23.6,20.8,18.0 ;{ }^{19} \mathrm{~F}-\mathrm{NMR}(282 \mathrm{MHz}$, $\left.\mathrm{CDCl}_{3}\right) \delta-80.5(\mathrm{t}, J=9.6 \mathrm{~Hz}, 3 \mathrm{~F}),-114.4$ (quint., $\left.2 \mathrm{~F}\right),-121.7(\mathrm{~m}, 6 \mathrm{~F}),-122.5(\mathrm{~s}, 2 \mathrm{~F}),-123.3$ (s, 2F), -125.9 (m, 2F) ; IR ( $\left.\mathrm{CHCl}_{3}\right): 3008,2929,1737,1497,1479,1454,1364,1278,1144$, $1086 \mathrm{~cm}^{-1}$; HRMS-MALDI Calcd for $\mathrm{C}_{117} \mathrm{H}_{135} \mathrm{~F}_{17} \mathrm{O}_{26} \mathrm{Na} 2301.8868(\mathrm{M}+\mathrm{Na})^{+}$; Found 2301.8814.

Pent-4(Z)-en-1-yl 6-O-\{6-O-[6-O-(3,4-di- $O$-benzyl-2- $O$-pivaloyl- $\beta$-D-glucopyranosyl)-3,4-di- $O$-benzyl-2- $O$-pivaloyl- $\beta$-D-glucopyranosyl]-3,4-di- $O$-benzyl-2- $O$-pivaloyl$\beta$-D-glucopyranosyl\}-3,4-di- $O$-benzyl-2- $O$-pivaloyl- $\beta$-D-glucopyranoside (13). To a solution of $11(4.7 \mathrm{mg}, 2.1 \mu \mathrm{mol})$ in $\mathrm{CH}_{2} \mathrm{Cl}_{2}(2 \mathrm{~mL})$ was added Grubbs' $2^{\text {nd }}$ generation catalyst $(0.2 \mathrm{mg}, 5 \mathrm{~mol} \%, 0.3 \mu \mathrm{mol})$ at room temperature. The solution was degassed with vacuum/argon (3 times) and ethylene (excess, $1 \mathrm{~atm}$.) was bubbled into the solution for one minute. The reaction mixture was stirred at room temperature under ethylene atmosphere for $12 \mathrm{~h}$, the TFT $(0.5 \mathrm{~mL})$ and Grubbs' catalyst $(0.2 \mathrm{mg})$ were added and ethylene was bubbled into the solution for another minute. After $12 \mathrm{~h}$ under ethylene atmosphere, Grubbs' catalyst $(0.2 \mathrm{mg})$ were added and ethylene was bubbled into the solution for $1 \mathrm{~min}$. After another $12 \mathrm{~h}$ under ethylene atmosphere, the black solution was evaporated to dryness in vacuo. The residue was purified using HPLC to afford $13\left(\mathrm{r}_{\mathrm{t}} 65 \mathrm{~min}, 2.1 \mathrm{mg}, 56 \%\right)$ and $11\left(\mathrm{r}_{\mathrm{t}} 69 \mathrm{~min}, 2.1\right.$ $\mathrm{mg}, 44 \%)$ as white solids. $\mathrm{R}_{\mathrm{f}} 0.41($ EtOAc/cyclohexane $=1: 3) ;[\alpha]_{\mathrm{D}}{ }^{\mathrm{rt}}=-14.4^{\circ}(\mathrm{c}=0.5$, $\mathrm{CHCl}_{3}$ ); ${ }^{1} \mathrm{H}-\mathrm{NMR}\left(300 \mathrm{MHz}, \mathrm{CDCl}_{3}\right) \delta$ 7.34-7.18 (m, 40H), 5.78 (dddd, $J=17.1,10.2,6.6$, $6.6 \mathrm{~Hz}, 1 \mathrm{H}), 5.10-4.92(\mathrm{~m}, 6 \mathrm{H}), 4.80-4.56(\mathrm{~m}, 16 \mathrm{H}), 4.50(\mathrm{~d}, J=11.0 \mathrm{~Hz}, 1 \mathrm{H}), 4.42$ (d, $J=7.7 \mathrm{~Hz}, 2 \mathrm{H}), 4.35(\mathrm{~d}, J=8.0 \mathrm{~Hz}, 1 \mathrm{H}), 4.02-3.30(\mathrm{~m}, 22 \mathrm{H}), 2.53(\mathrm{t}, J=6.3,6.3 \mathrm{~Hz}, 1 \mathrm{H})$, 2.12-2.02 (m, 2H), 1.69-1.58 (m, 2H), 1.19 (s, 9H), 1.19 (s, 9H), 1.18 (s, 9H), $1.16(\mathrm{~s}, 9 \mathrm{H})$;

${ }^{13} \mathrm{C}-\mathrm{NMR}\left(75 \mathrm{MHz}, \mathrm{CDCl}_{3}\right) \delta$ 176.7, 176.6, 176.4, 138.1, 138.0, 138.0, 137.9, 137.8, 137.7, $128.4,128.4,128.3,128.2,128.0,127.9,127.9,127.8,127.5,127.3,127.2,114.9,102.0$, $101.7,100.8,83.3,83.2,83.0,78.5,77.8,77.3,75.8,75.3,75.1,74.9,74.8,74.7,74.5,73.2$, 
73.1, 72.7, 69.4, 69.1, 68.9, 67.9, 61.9, 38.9, 30.2, 28.9, 27.1, 27.1; IR $\left(\mathrm{CHCl}_{3}\right): 3064,3038$, 2966, 2875, 1737, 1605, 1497, 1479, 1454, 1397, 1363, 1278, 1261, 1200, 1139, 1086, 1038, $914 \mathrm{~cm}^{-1}$; HRMS-MALDI Calcd for $\mathrm{C}_{105} \mathrm{H}_{130} \mathrm{O}_{25} \mathrm{Na} 1813.8793(\mathrm{M}+\mathrm{Na})^{+}$; Found: 1813.9023.

$O$-(6-O-\{6-O-[6-O-(6-O-Acetyl-3,4-di- $O$-benzyl-2- $O$-pivaloyl- $\beta$-D-glucopyranosyl)3,4-di- $O$-benzyl-2- $O$-pivaloyl- $\beta$-D-glucopyranosyl]-3,4-di- $O$-benzyl-2- $O$-pivaloyl- $\beta$-Dglucopyranosyl\}-3,4-di- $O$-benzyl-2- $O$-pivaloyl- $\alpha$-D-glucopyranosyl)trichloroacetimidate (14). To a solution of $12(14.2 \mathrm{mg}, 6.23 \mu \mathrm{mol})$ in $\mathrm{CH}_{3} \mathrm{CN}\left(+1 \% \mathrm{H}_{2} \mathrm{O}, 630 \mu \mathrm{L}\right)$ was added NBS $(3.3 \mathrm{mg}, 18.7 \mathrm{mmol}$ ) at room temperature. After $30 \mathrm{~min}$, the solution was treated with saturated aqueous $\mathrm{Na}_{2} \mathrm{~S}_{2} \mathrm{O}_{3}$ solution $(0.6 \mathrm{~mL})$ and water $(0.6 \mathrm{~mL})$ and extracted with $\mathrm{Et}_{2} \mathrm{O}$ ( $3 \times 5 \mathrm{~mL}$ ). The combined organic phase was dried over $\mathrm{MgSO}_{4}$ and the solvents were evaporated in vacuo to afford the crude lactol [(23 mg, $\alpha / \beta \sim 1: 1, \mathrm{R}_{\mathrm{f}(\alpha)} 0.27, \mathrm{R}_{\mathrm{f}(\beta)} 0.15$ $($ EtOAc/cyclohexane $=1: 3)]$ as yellowish oil. The residue was dissolved in $\mathrm{CH}_{2} \mathrm{Cl}_{2}(600 \mu \mathrm{L})$ and trichloroacetonitrile $(20 \mu \mathrm{L}, 32 \mathrm{eq}, 0.2 \mathrm{mmol})$ was added at room temperature. The solution was cooled to $0{ }^{\circ} \mathrm{C}$ and $\mathrm{DBU}(1 \mu \mathrm{L}, 6.7 \mu \mathrm{mol})$ was added. After $10 \mathrm{~min}$, the solution was allowed to warm to room temperature and was stirred for further $30 \mathrm{~min}$. The solvents were evaporated in vacuo and the residue was purified by silica gel flash column chromatography $\left(\right.$ EtOAc/cyclohexane $\left.=1: 4,+1 \% \mathrm{NEt}_{3}\right)$ to afford $14(11.7 \mathrm{mg}, 98 \%, \alpha / \beta=$ 6:1) as colorless oil. $\mathrm{R}_{\mathrm{f}(\alpha)} 0.35$ (EtOAc/cyclohexane $\left.=1: 4\right) ;[\alpha]_{\mathrm{D}}{ }^{\mathrm{rt}}=4.7^{\circ}\left(c=0.5, \mathrm{CHCl}_{3}\right)$; ${ }^{1} \mathrm{H}-\mathrm{NMR}\left(300 \mathrm{MHz}, \mathrm{CDCl}_{3}, \alpha\right.$-isomer) $\delta 8.67(\mathrm{~s}, 1 \mathrm{H}), 7.35-7.19(\mathrm{~m}, 40 \mathrm{H}), 6.50(\mathrm{~d}$, $J=3.6 \mathrm{~Hz}, 1 \mathrm{H}), 5.11(\mathrm{dd}, J=9.1,7.7 \mathrm{~Hz}, 1 \mathrm{H}), 5.09$ (dd, $J=9.3,7.1 \mathrm{~Hz}, 1 \mathrm{H}), 5.07$ (dd, $J=10.2,3.6 \mathrm{~Hz}, 1 \mathrm{H}), 5.01(\mathrm{dd}, J=8.5,8.3 \mathrm{~Hz}, 1 \mathrm{H}), 4.82-4.46(\mathrm{~m}, 19 \mathrm{H}), 4.30(\mathrm{dd}, J=11.2$, $2.2 \mathrm{~Hz}, 1 \mathrm{H}), 4.16(\mathrm{dd}, J=12.1,5.1 \mathrm{~Hz}, 1 \mathrm{H}), 4.13-3.42(\mathrm{~m}, 18 \mathrm{H}), 1.93$ (s, 3H), 1.17 (s, 9H), 1.15 (s, 9H), 1.14 (s, 9H), $1.10(\mathrm{~s}, 9 \mathrm{H})$; IR $\left(\mathrm{CHCl}_{3}\right): 3038,2965,2872,1737,1674,1602$, 1497, 1479, 1455, 1397, 1363, 1262, 1200, 1138, 1090, $910 \mathrm{~cm}^{-1}$; HRMS-MALDI Calcd for $\mathrm{C}_{104} \mathrm{H}_{124} \mathrm{Cl}_{3} \mathrm{NO}_{26} \mathrm{Na} 1930.7369(\mathrm{M}+\mathrm{Na})^{+}$; Found: 1930.7317.

4-Oxobutan-1-yl 6-O-\{6-O-[6-O-(6-O-acetyl-3,4-di- $O$-benzyl-2-O-pivaloyl- $\beta$-Dglucopyranosyl)-3,4-di- $O$-benzyl-2- $O$-pivaloyl- $\beta$-D-glucopyranosyl]-3,4-di- $O$-benzyl-2- $O$ pivaloyl- $\beta$-D-glucopyranosyl\}-3,4-di- $O$-benzyl-2- $O$-pivaloyl- $\beta$-D-glucopyranoside

To a solution of $12(12.0 \mathrm{mg}, 5.3 \mu \mathrm{mol})$ in $\mathrm{CH}_{2} \mathrm{Cl}_{2}(1.5 \mathrm{~mL})$ was bubbled $\mathrm{O}_{3}\left(3 \%\right.$ in $\left.\mathrm{O}_{2}\right)$ at -78 ${ }^{\circ} \mathrm{C}$ for $3 \mathrm{~min}$. The light-blue solution was treated with $\mathrm{PPh}_{3}(2 \mathrm{mg}, 7.6 \mu \mathrm{mol})$ and was allowed to warm to room temperature. After $3 \mathrm{~h}$, the solution was treated with saturated aqueous 
$\mathrm{NH}_{4} \mathrm{Cl}$ solution $(5 \mathrm{~mL})$ and extracted with $\mathrm{CH}_{2} \mathrm{Cl}_{2}(3 \times 5 \mathrm{~mL})$. The combined organic phase was washed with brine $(3 \mathrm{~mL})$ and dried over $\mathrm{MgSO}_{4}$. The solvents were evaporated in vacuo and the residue was purified by silica gel flash column chromatography (EtOAc/hexane $=1: 3$ ) to afford $15(6.5 \mathrm{mg}, 68 \%)$ as colorless oil. $\mathrm{R}_{\mathrm{f}} 0.32($ EtOAc/hexane $=1: 3) ;[\alpha]_{\mathrm{D}}{ }^{\mathrm{rt}}=-13.3^{\circ}$ $\left(\mathrm{c}=0.61, \mathrm{CHCl}_{3}\right) ;{ }^{1} \mathrm{H}-\mathrm{NMR}\left(300 \mathrm{MHz}, \mathrm{CDCl}_{3}\right) \delta 9.76(\mathrm{t}, J=1.2 \mathrm{~Hz}, 1 \mathrm{H}), 7.32-7.14(\mathrm{~m}$, $40 \mathrm{H}), 5.10$ (dd, $J=7.8,6.8 \mathrm{~Hz}, 1 \mathrm{H}), 5.07$ (dd, $J=7.7,6.2 \mathrm{~Hz}, 1 \mathrm{H}), 5.03$ (dd, $J=7.8,7.8 \mathrm{~Hz}$, $1 \mathrm{H}), 5.00(\mathrm{dd}, J=8.1,8.1 \mathrm{~Hz}, 1 \mathrm{H}), 4.76-4.53(\mathrm{~m}, 16 \mathrm{H}), 4.44-4.22$ (m, 5H), 4.18 (dd, $J=11.8,5.0 \mathrm{~Hz}, 1 \mathrm{H}), 3.98-3.34(\mathrm{~m}, 20 \mathrm{H}), 2.57-2.48(\mathrm{~m}, 2 \mathrm{H}), 2.00(\mathrm{~s}, 3 \mathrm{H}), 1.91-1.80(\mathrm{~m}$, 2H), $1.20(\mathrm{~s}, 9 \mathrm{H}), 1.18(\mathrm{~s}, 9 \mathrm{H}), 1.18(\mathrm{~s}, 9 \mathrm{H}), 1.17(\mathrm{~s}, 9 \mathrm{H}),{ }^{13} \mathrm{C}-\mathrm{NMR}\left(75 \mathrm{MHz}, \mathrm{CDCl}_{3}\right) \delta$ 201.9; 176.6, 176.2, 170.4, 138.1, 138.0, 137.8, 137.7, 137.5, 128.3, 128.2, 128.2, 128.2, $127.8,127.5,127.3,127.2,127.0,101.6,101.0,100.5,83.1,77.2,74.9,74.6,74.2,73.2,73.0$ 69.1, 68.5, 63.4, 40.6, 38.9, 29.7, 27.3, 27.2, 22.2, 21.0; IR $\left(\mathrm{CHCl}_{3}\right): 3008,2960,1737,1455$, 1364, 1278, 1139, 1067, $909 \mathrm{~cm}^{-1}$; HRMS-MALDI Calcd for $\mathrm{C}_{106} \mathrm{H}_{130} \mathrm{O}_{27} \mathrm{Na} 1857.8697$ $(\mathrm{M}+\mathrm{Na})^{+}$; Found: 1857.8649 .

\section{References}

(1) Carrel, F.; Seeberger, P.H. J. Carbohydr. Chem., 2007, in press.

(2) Markidis, T.; Kokotos, G. J. Org. Chem., 2002, 67, 1685-1688.

(3) Rocaboy, C.; Bauer, W.; Gladysz, J. A. Eur. J. Org. Chem., 2000, 14, 2621-2628. 

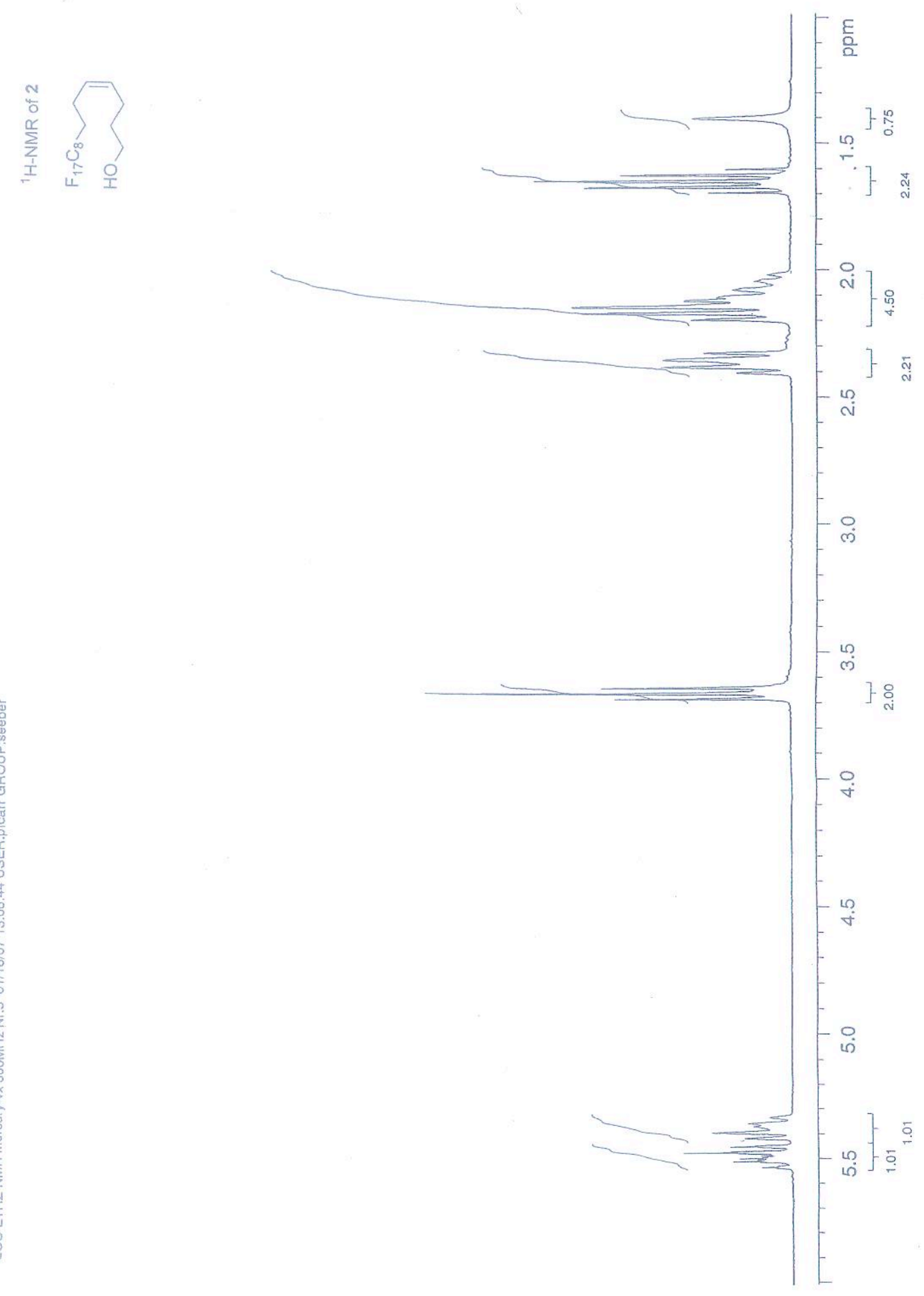


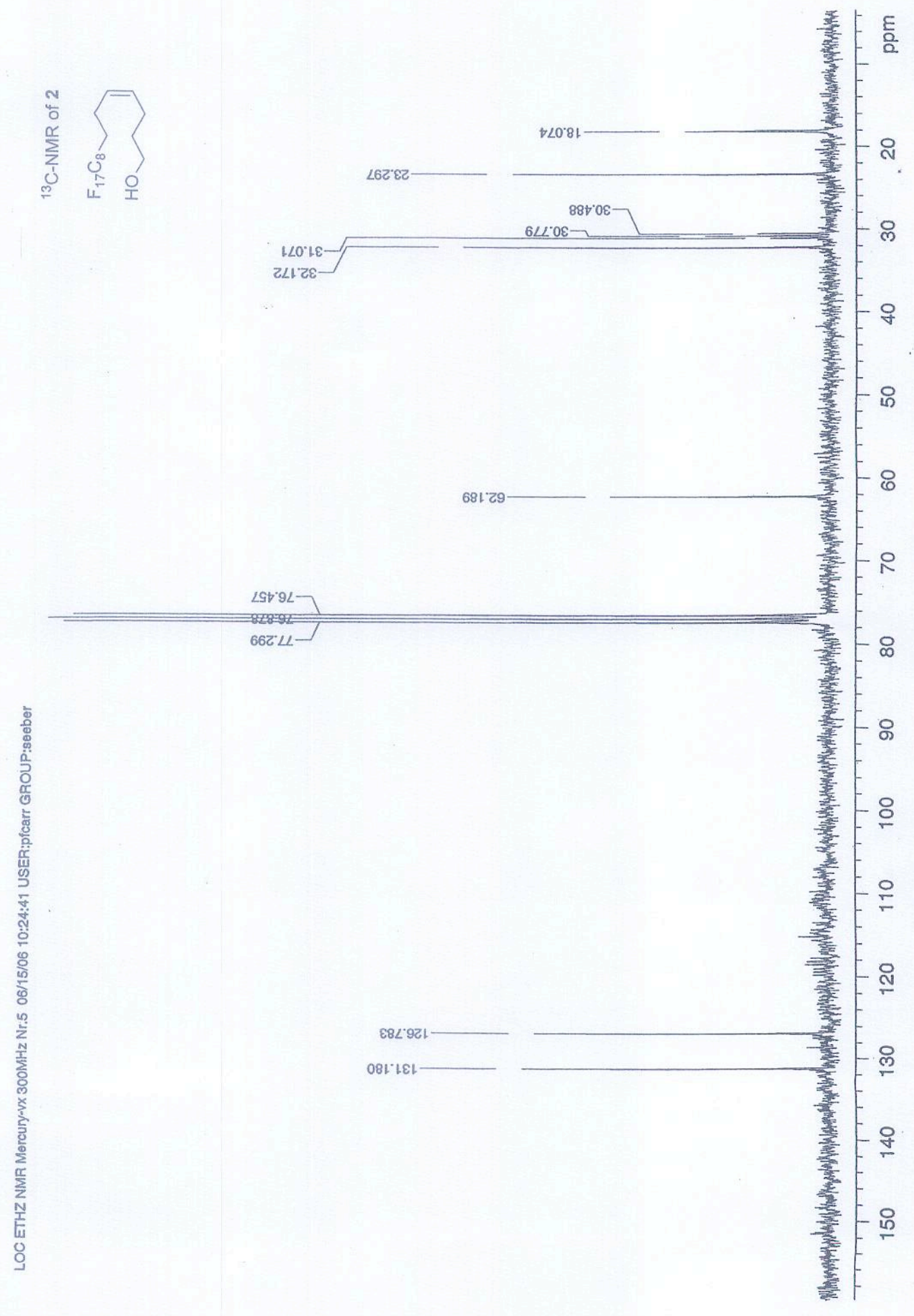




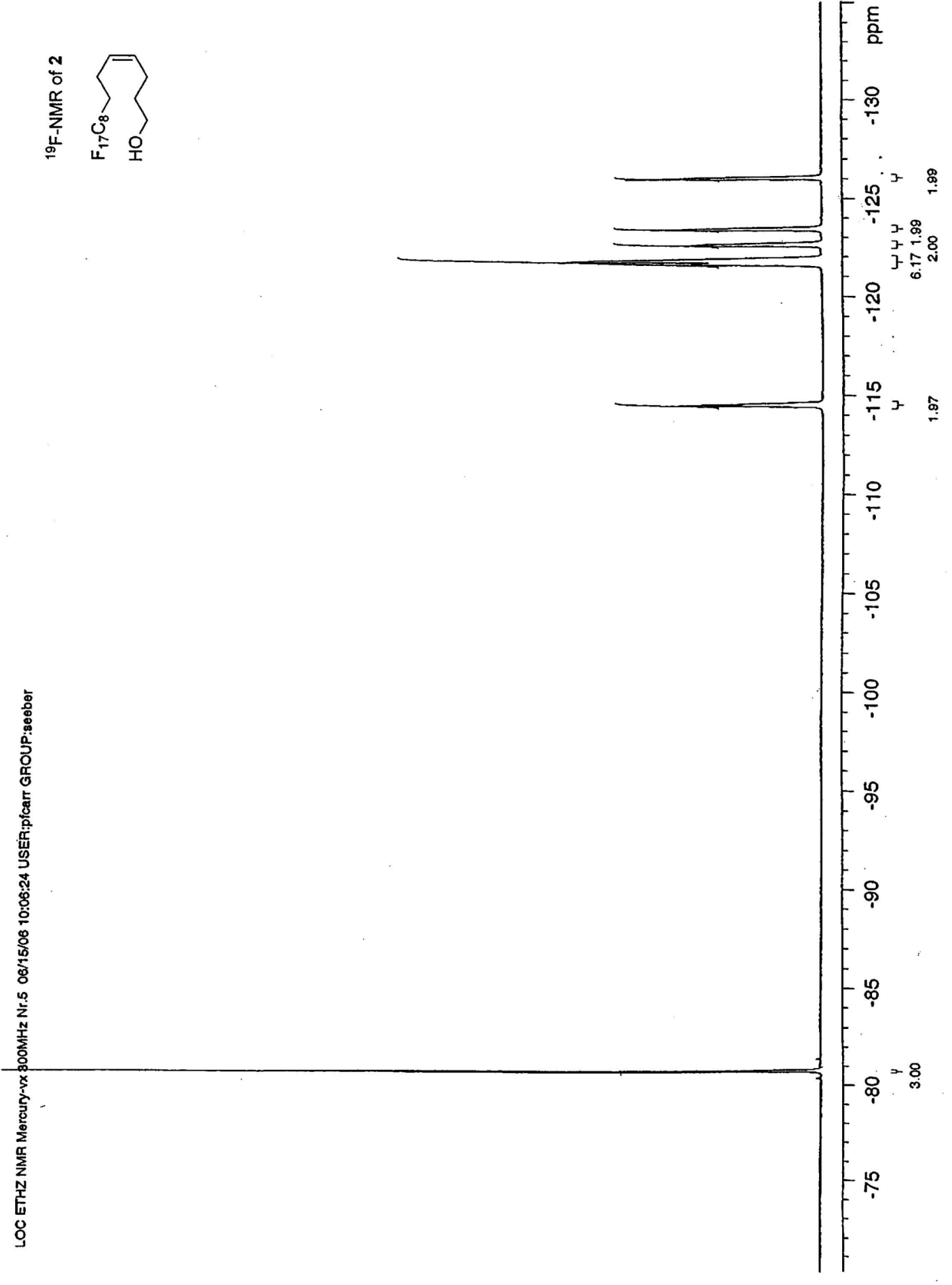




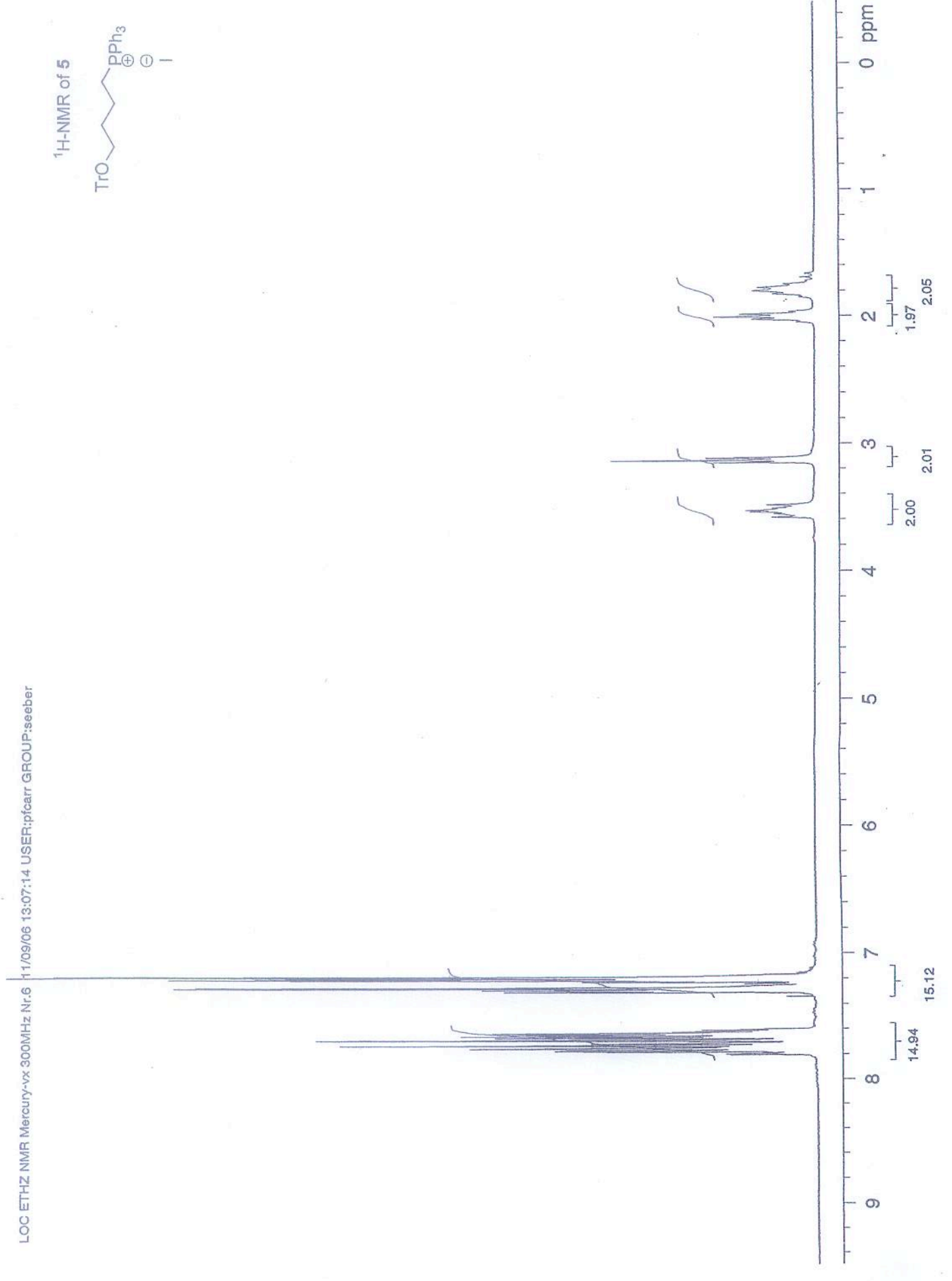




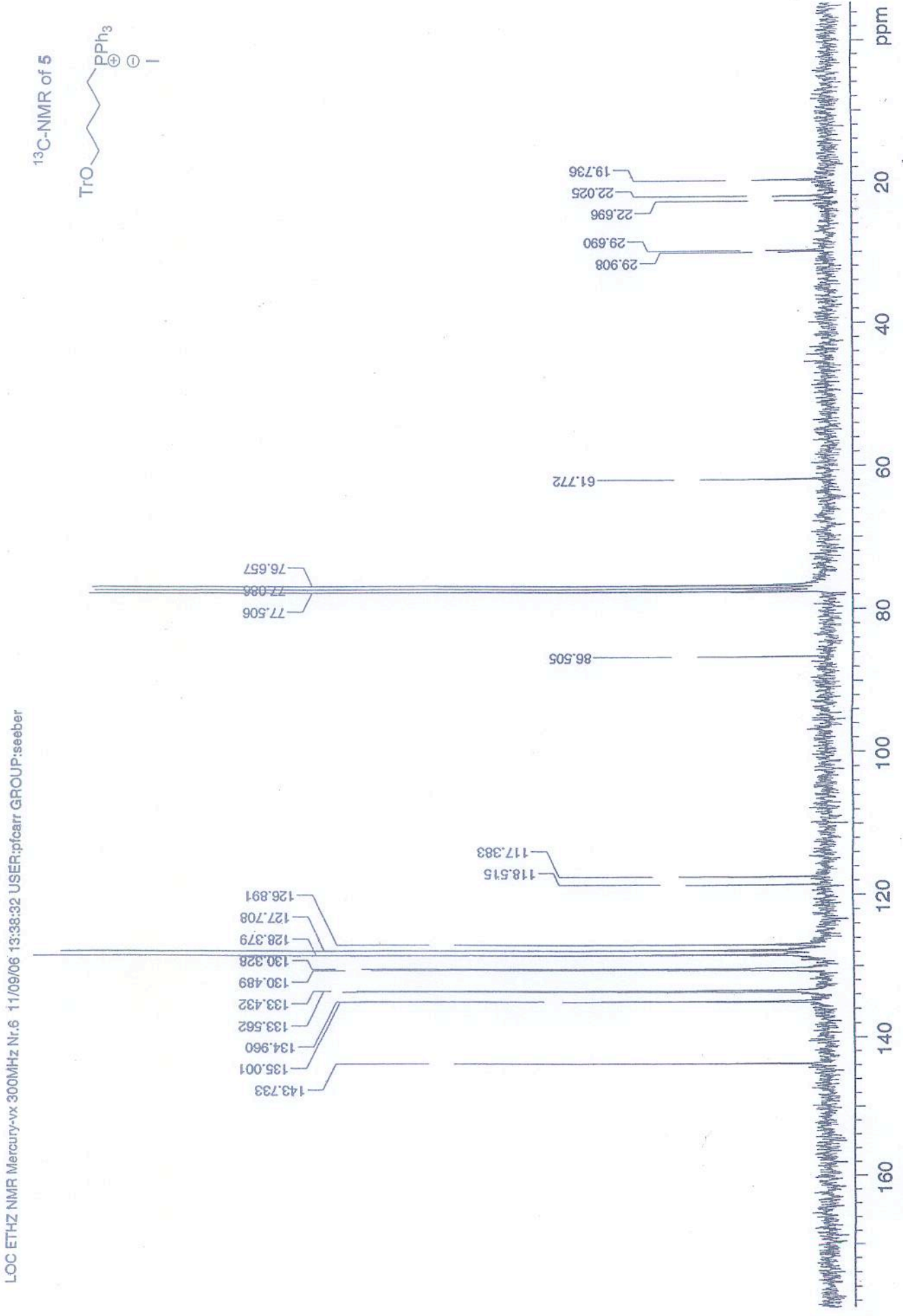




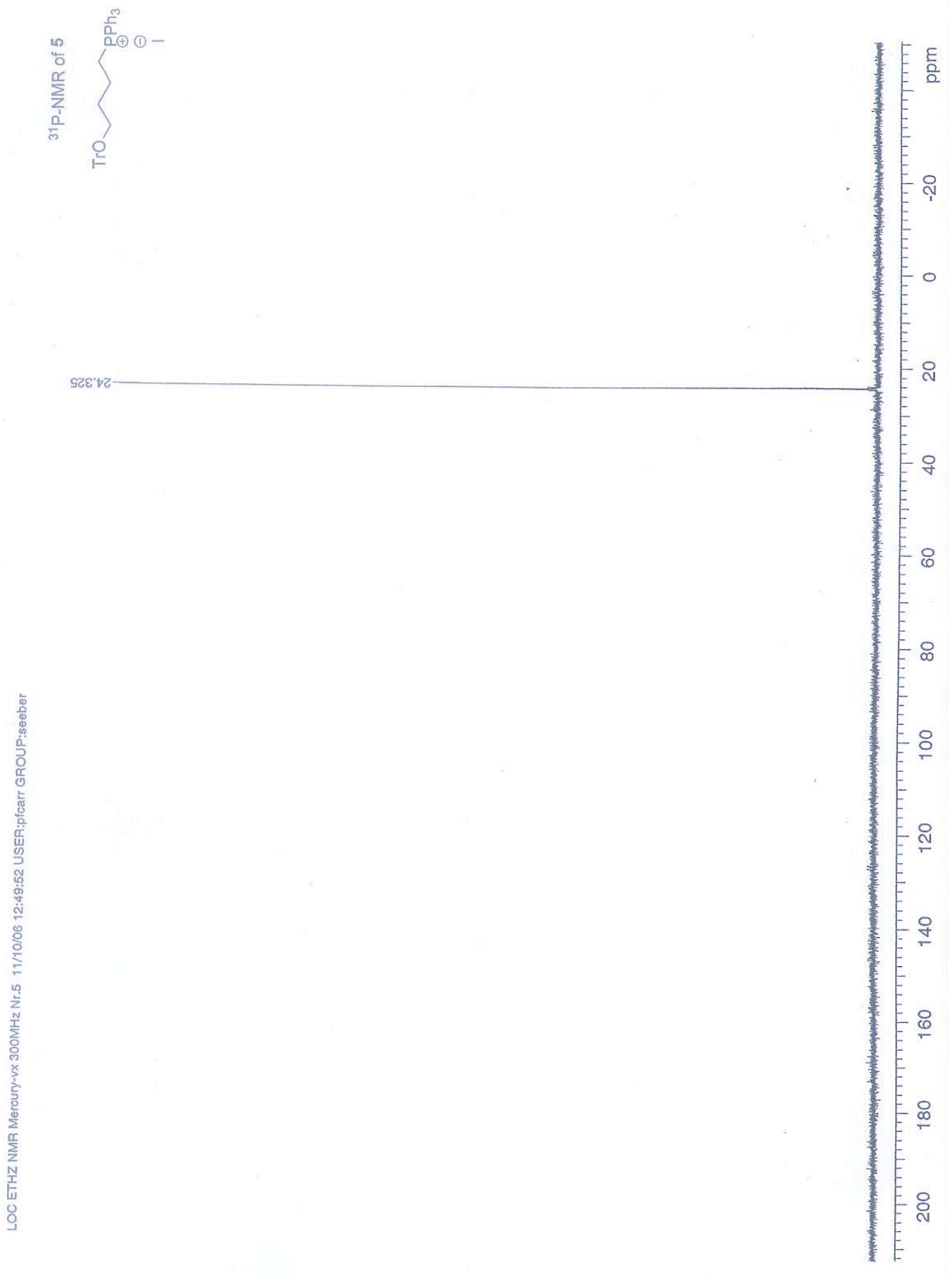



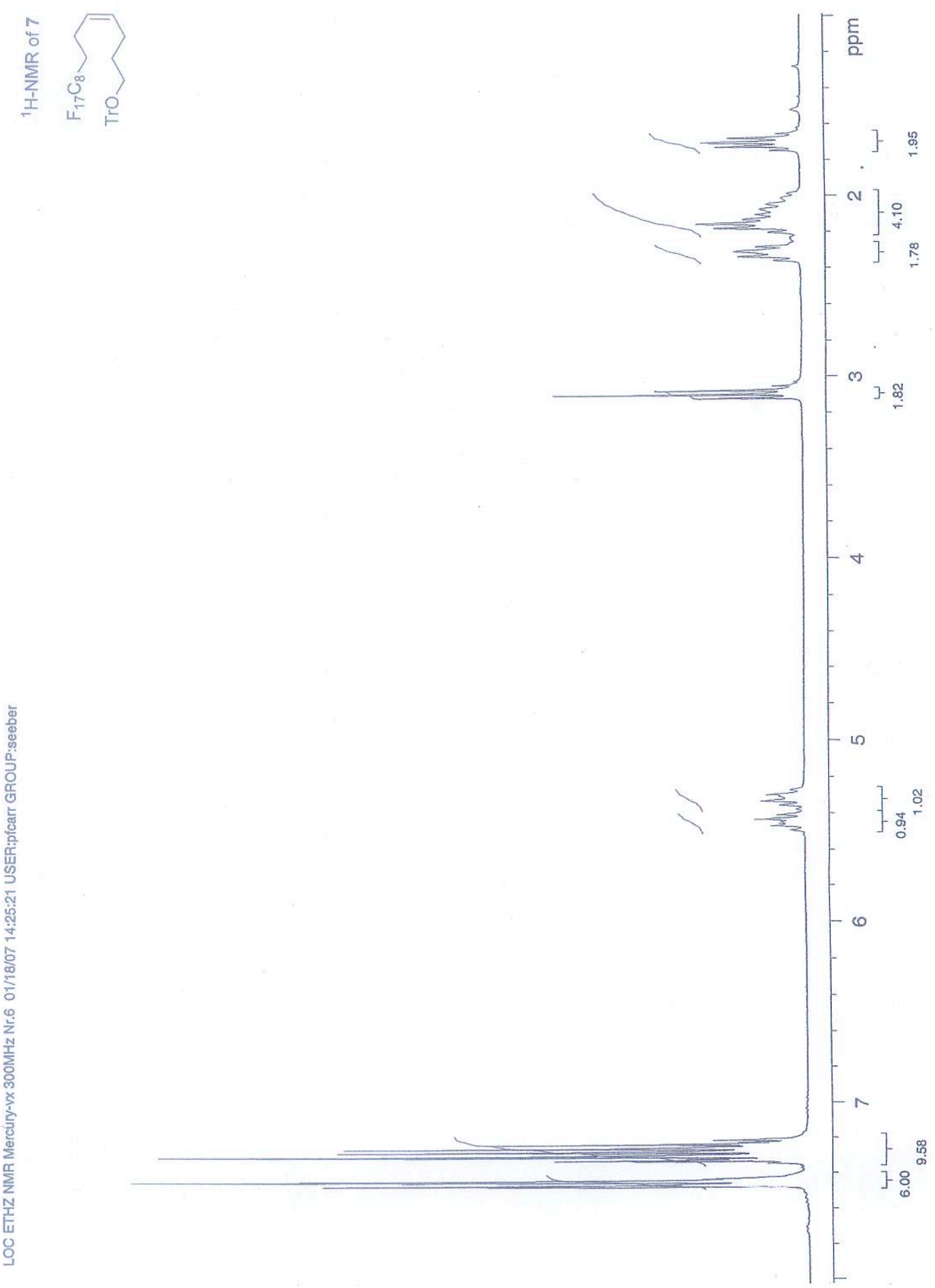

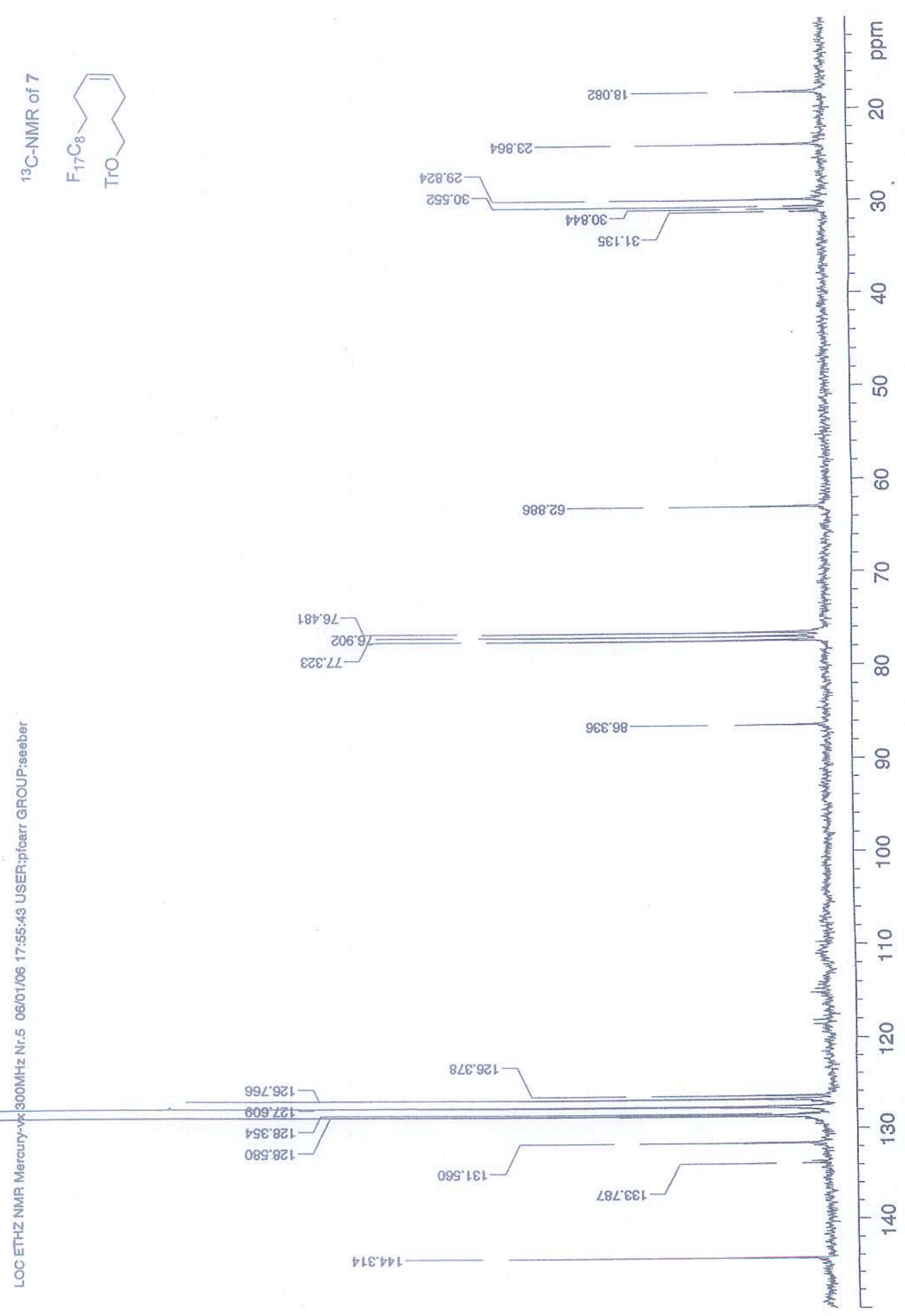


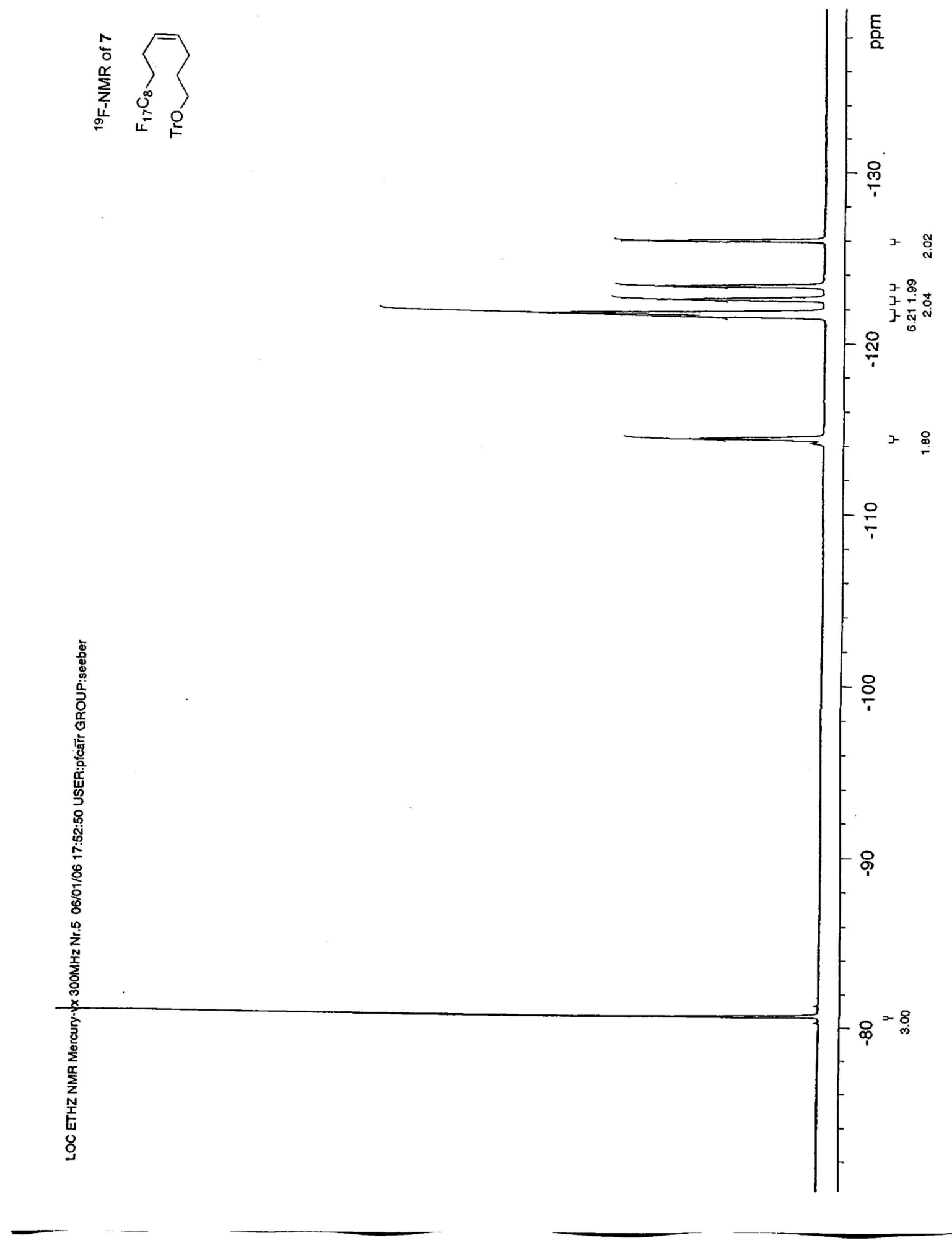

S22 


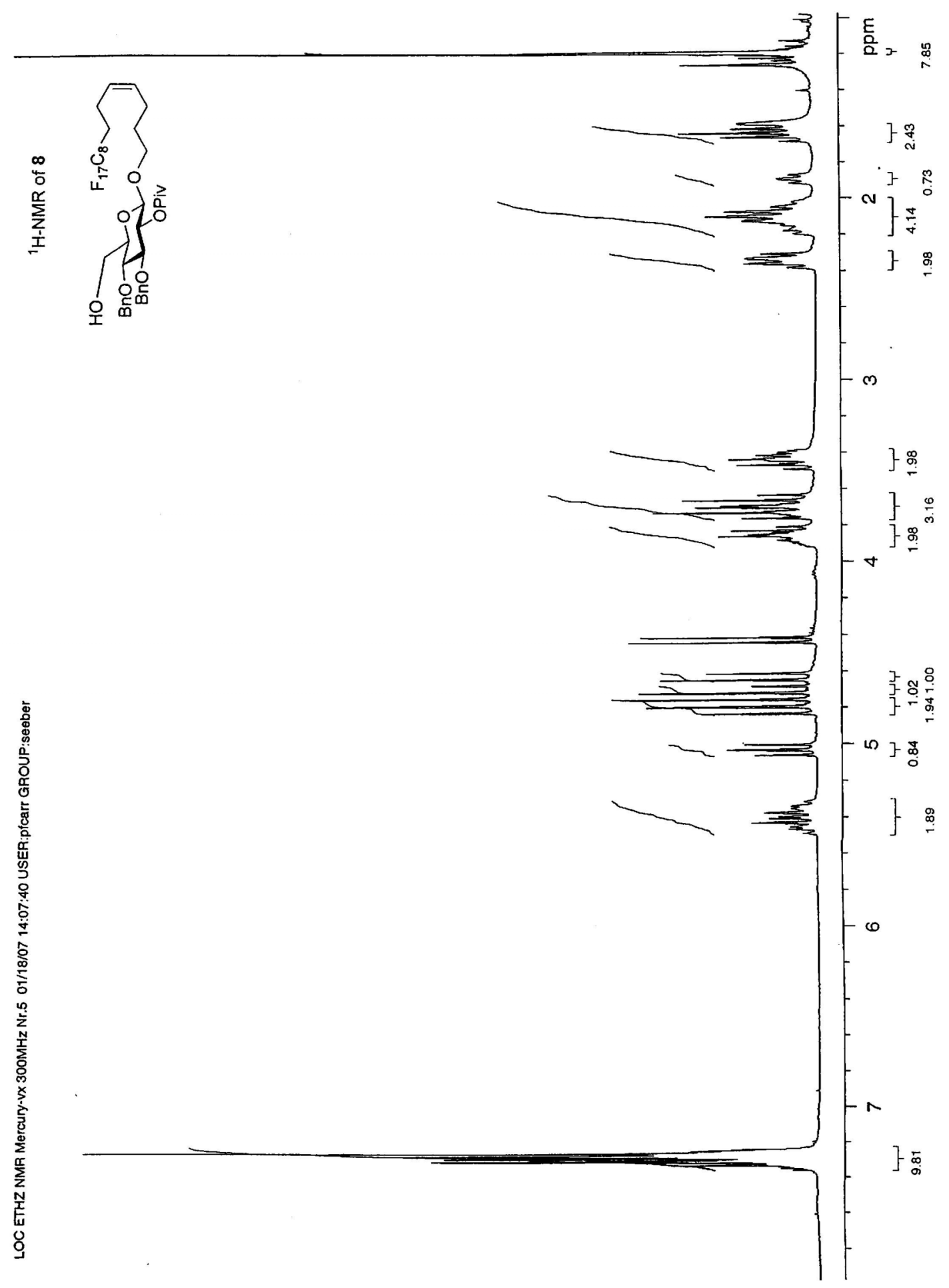




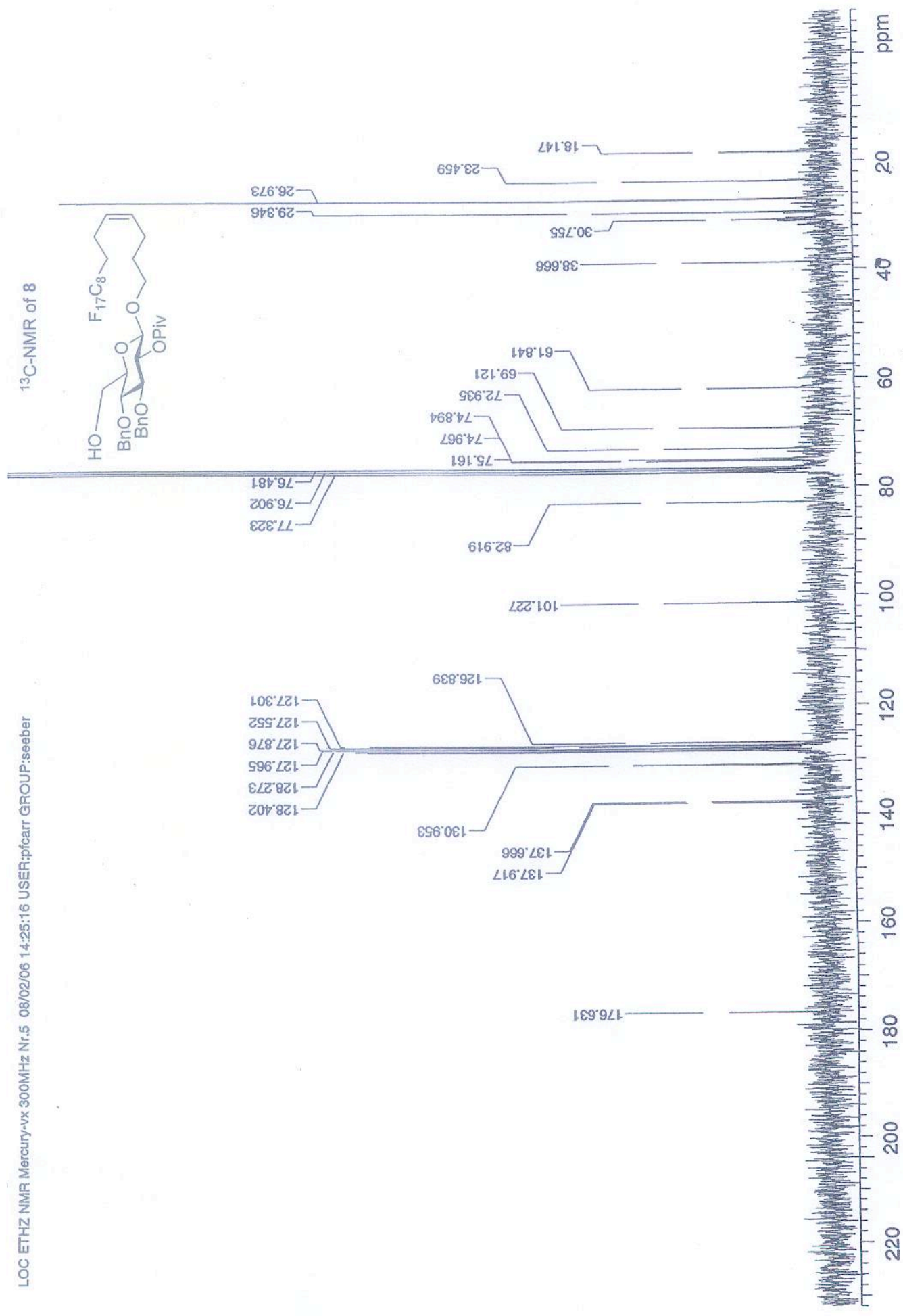



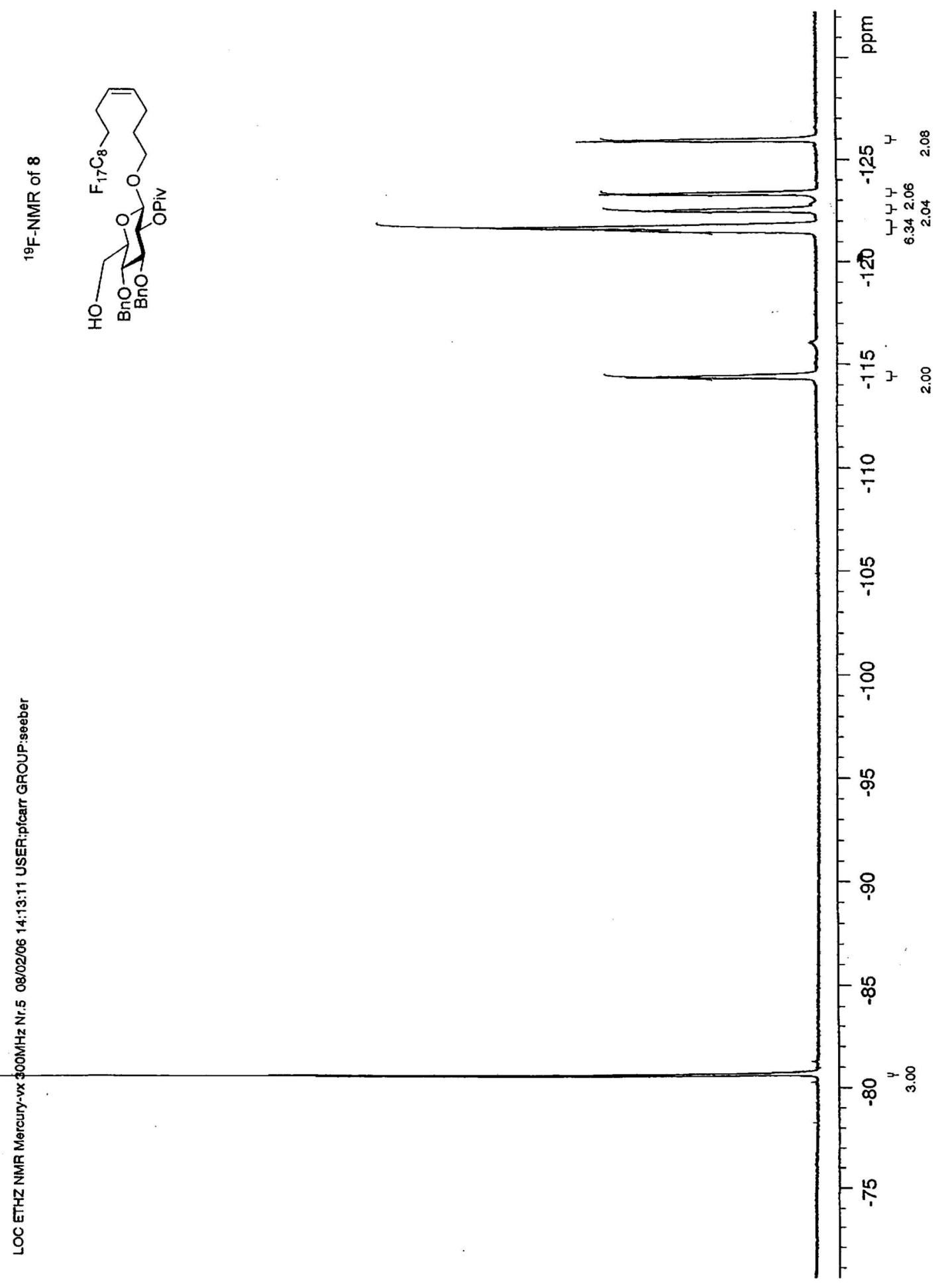


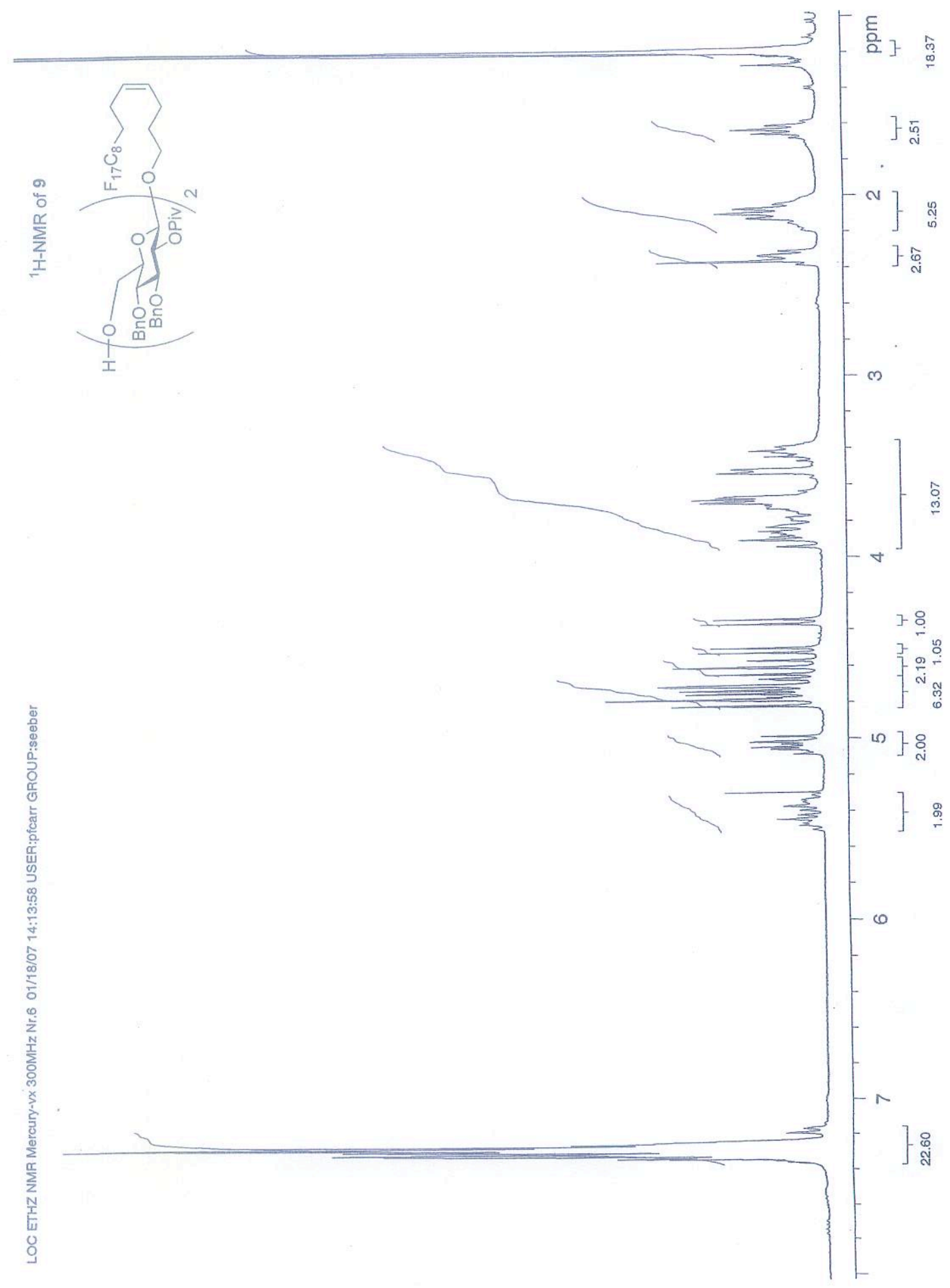




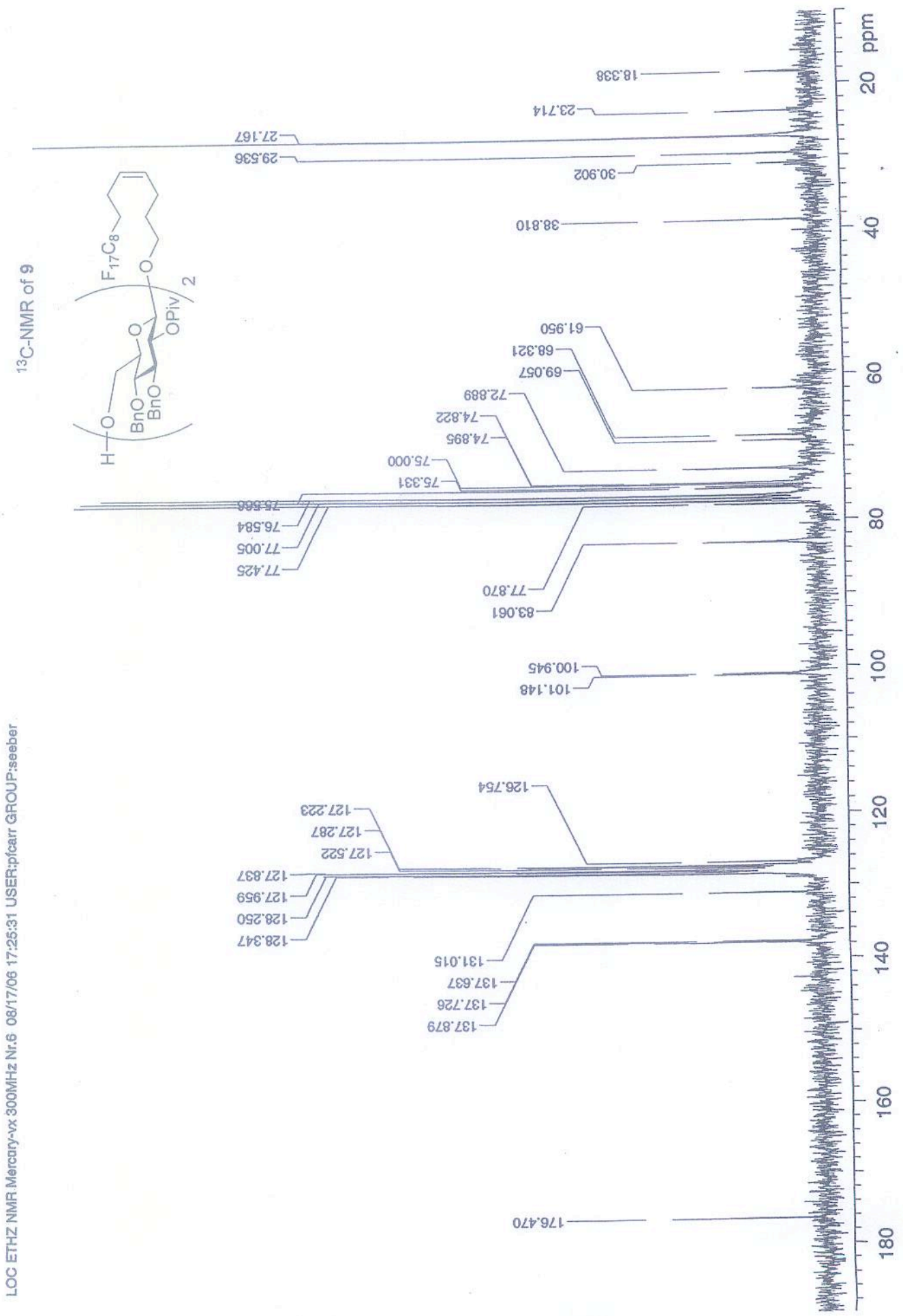



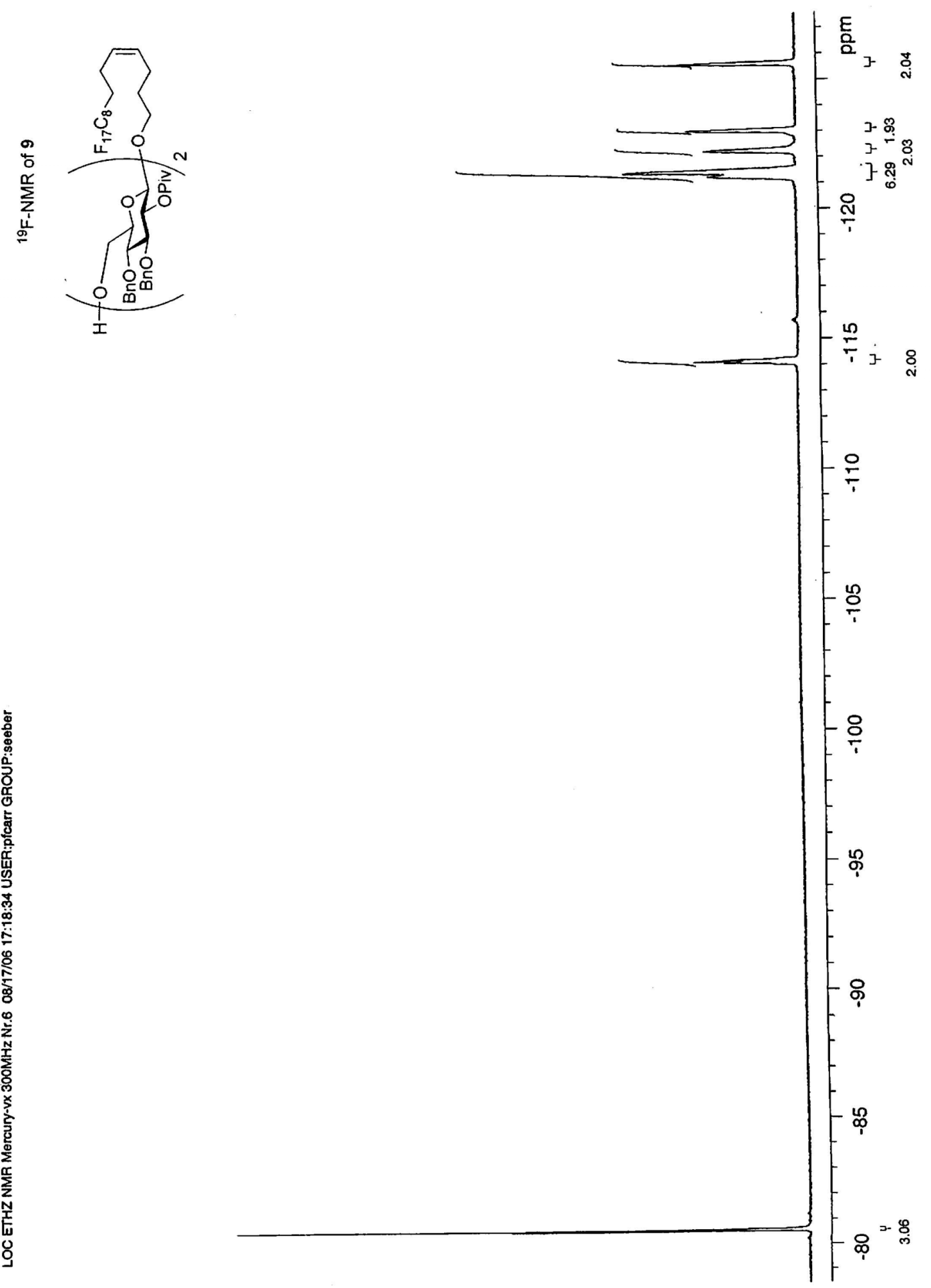


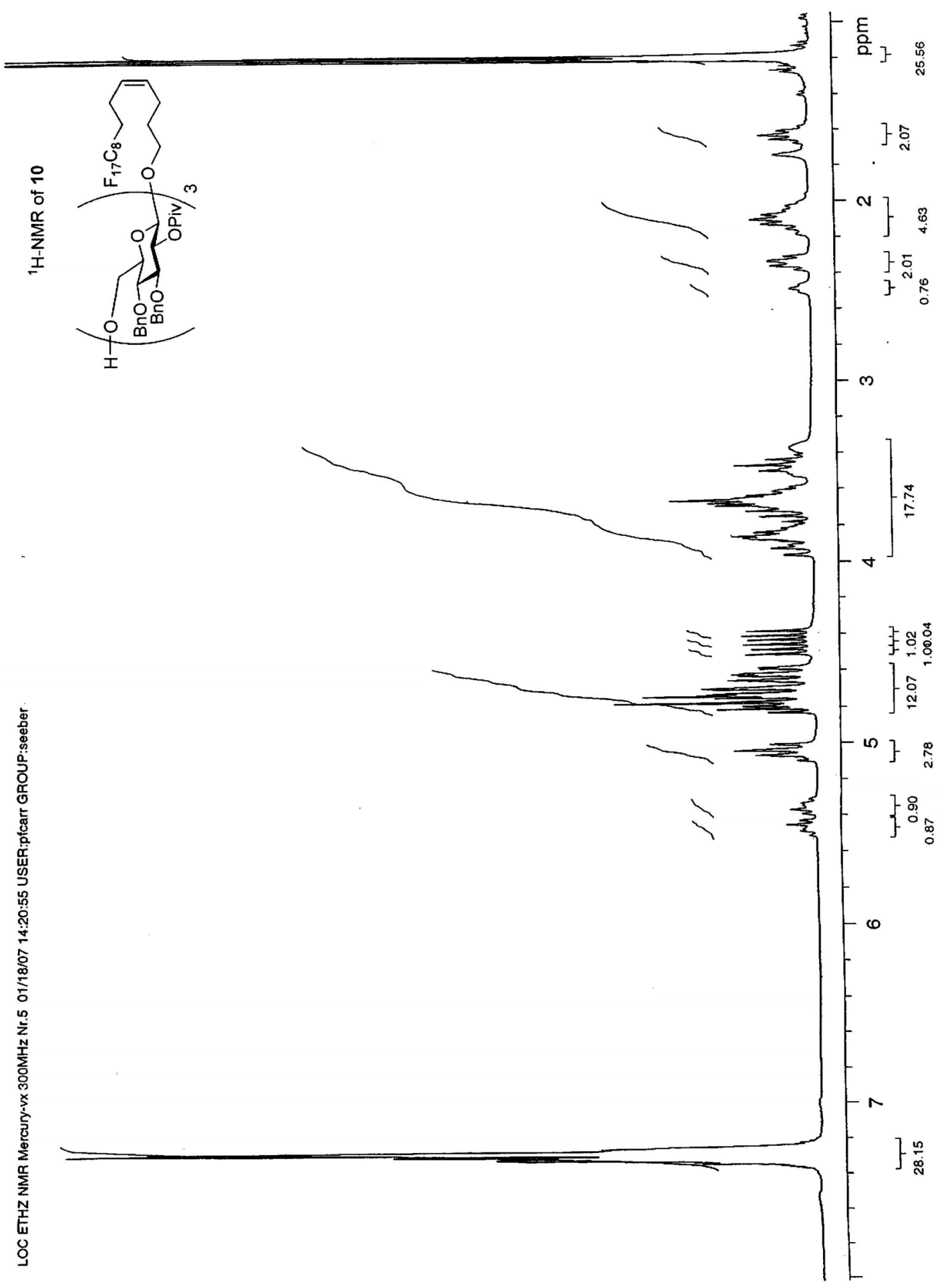




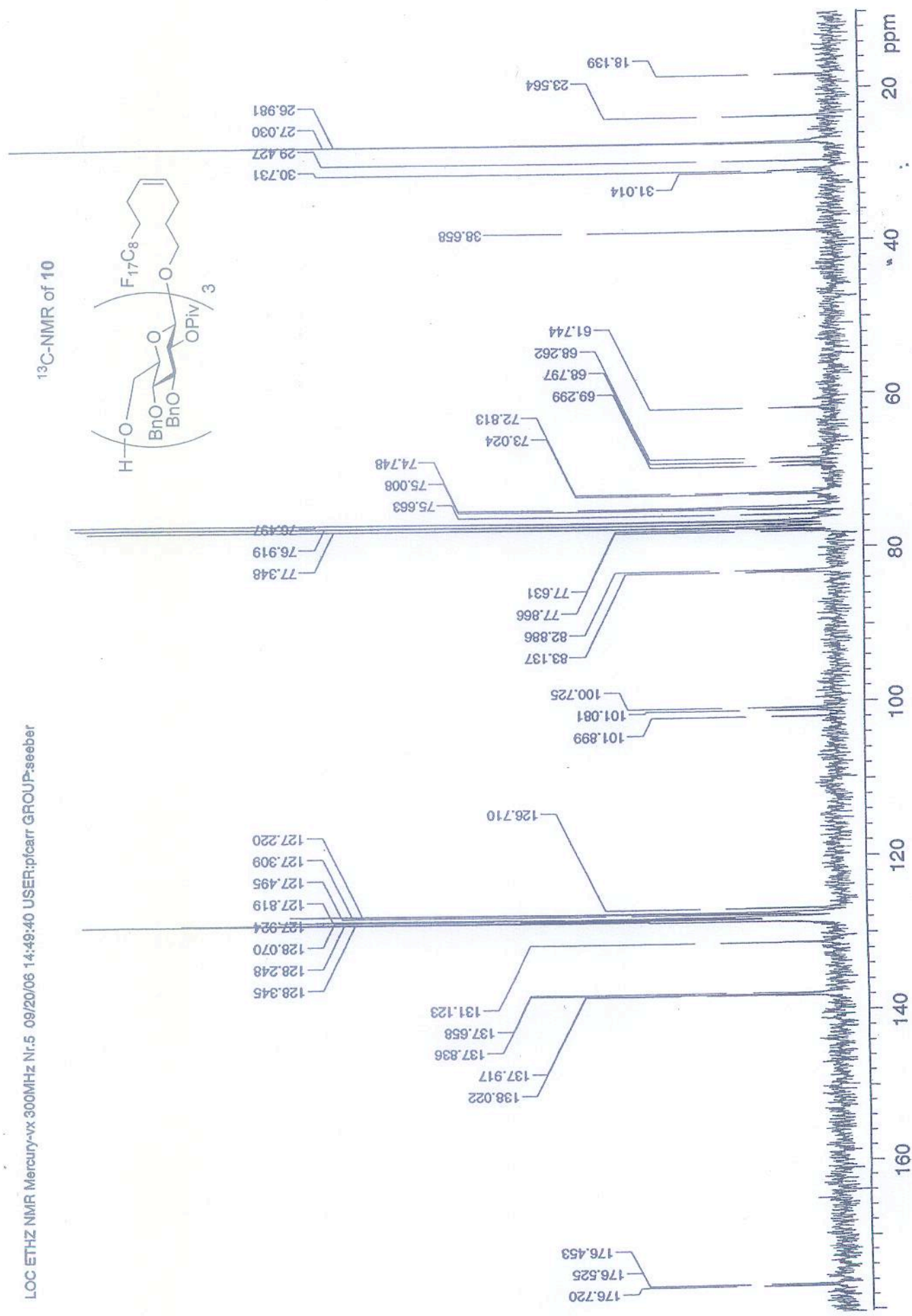



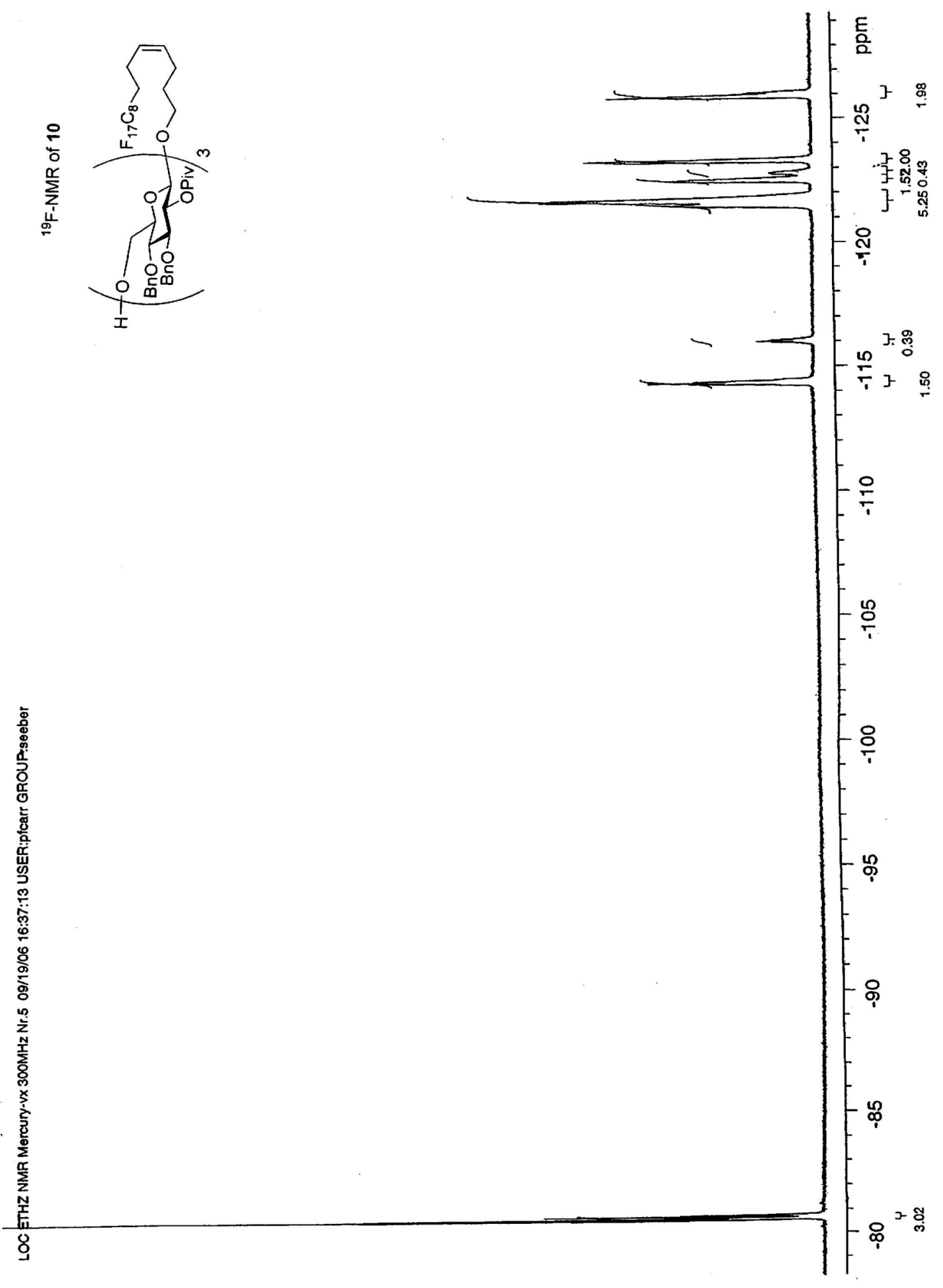

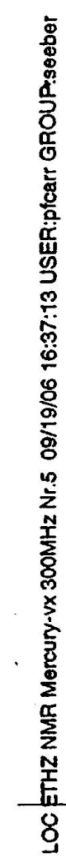




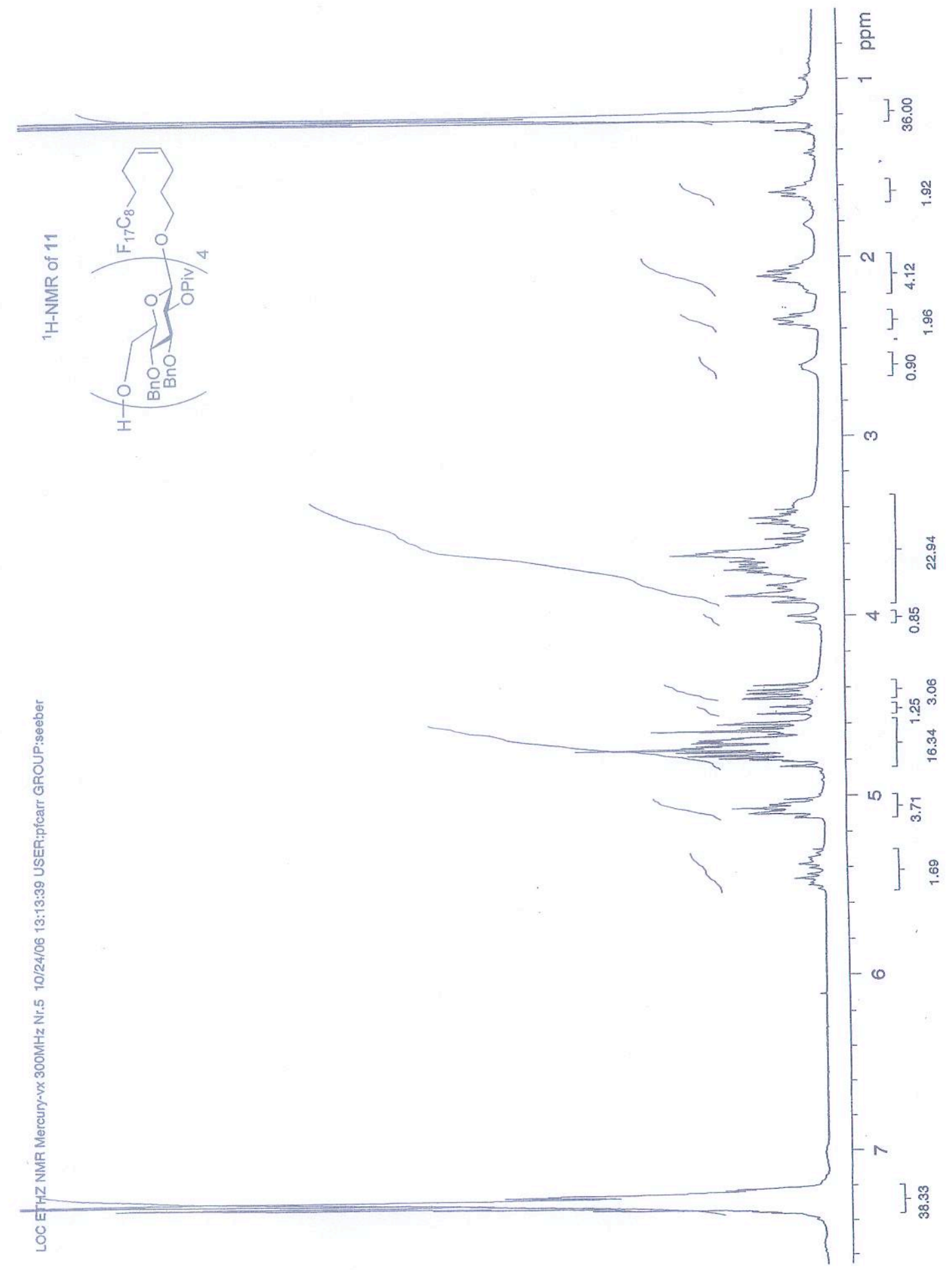




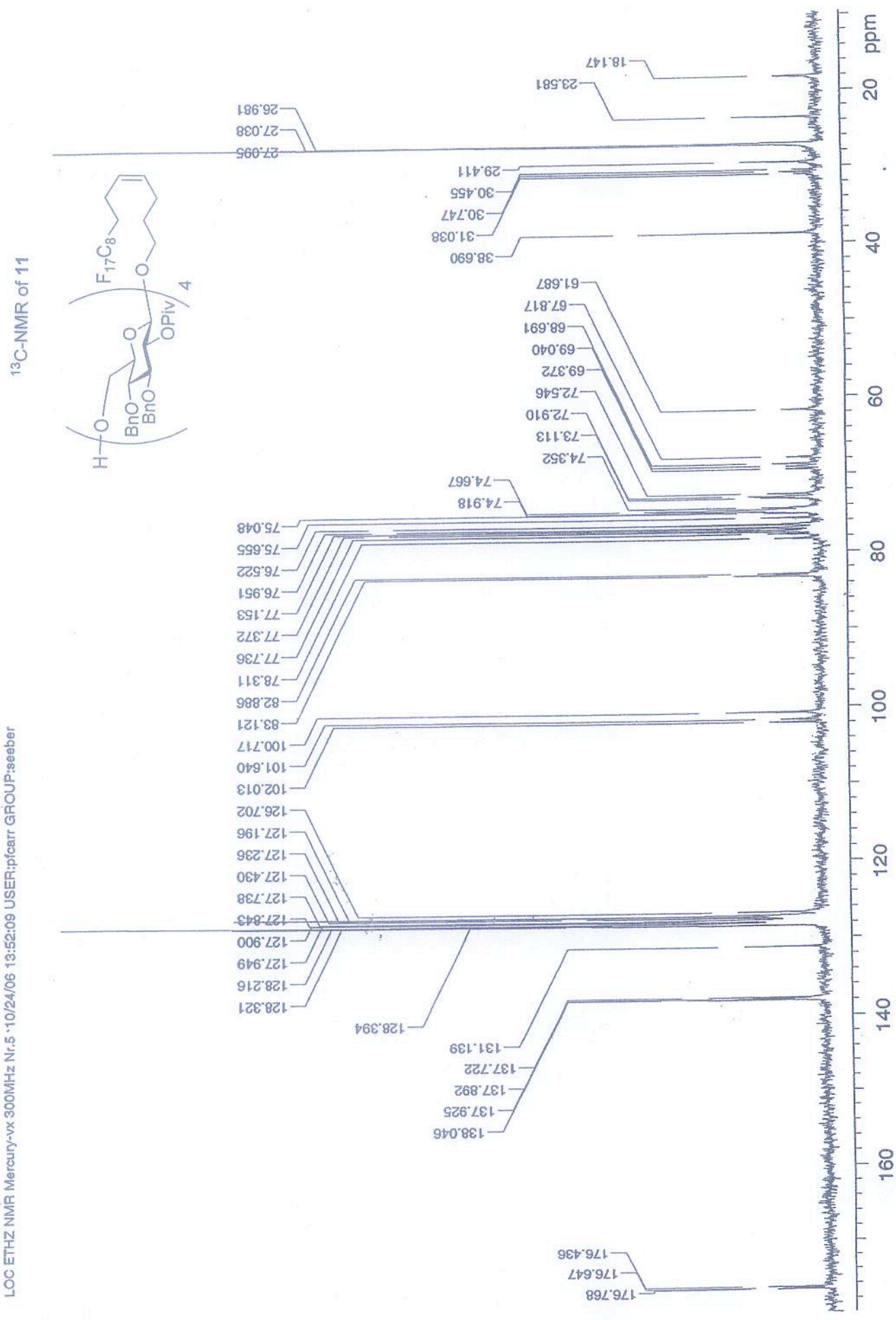



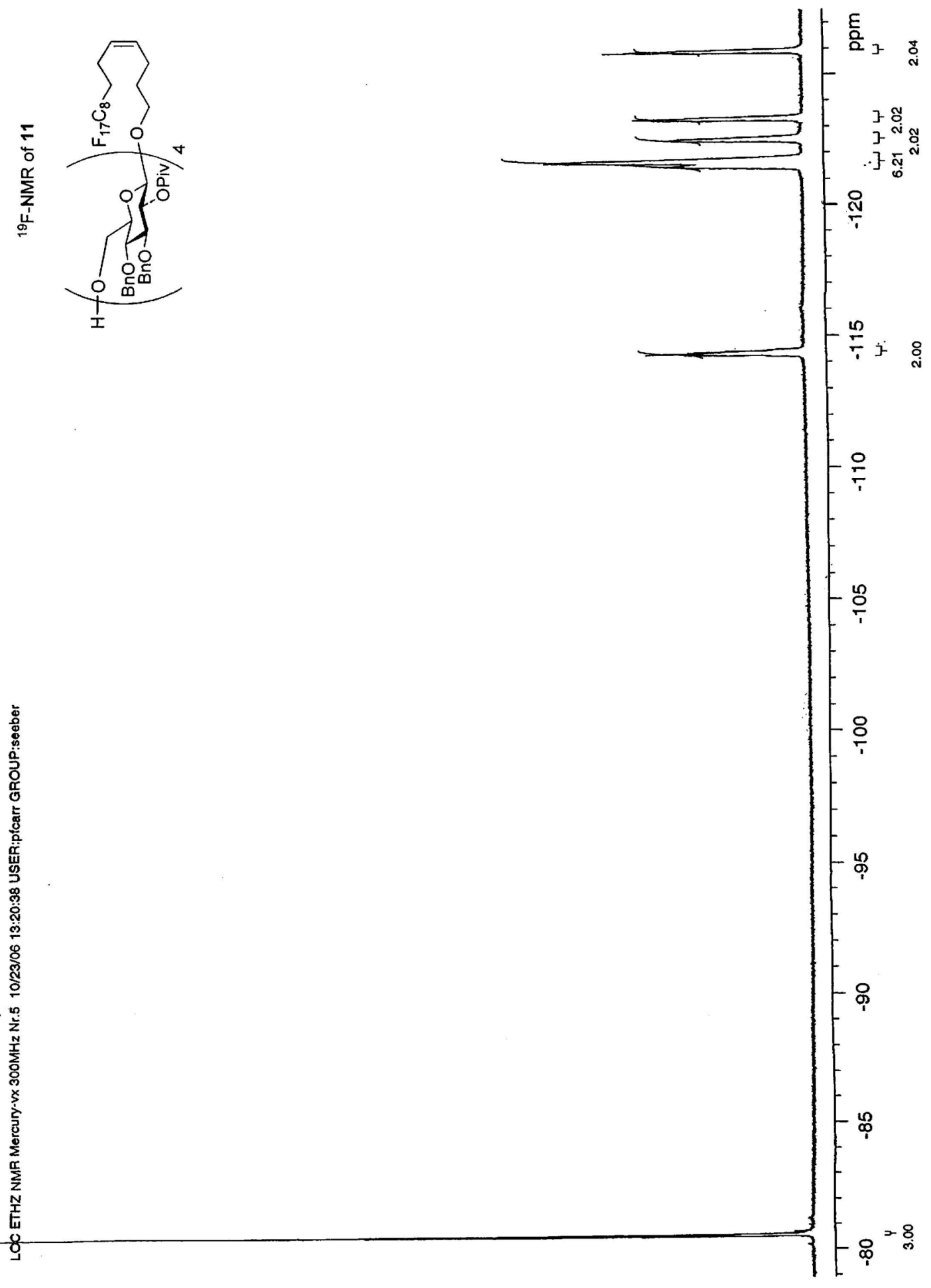


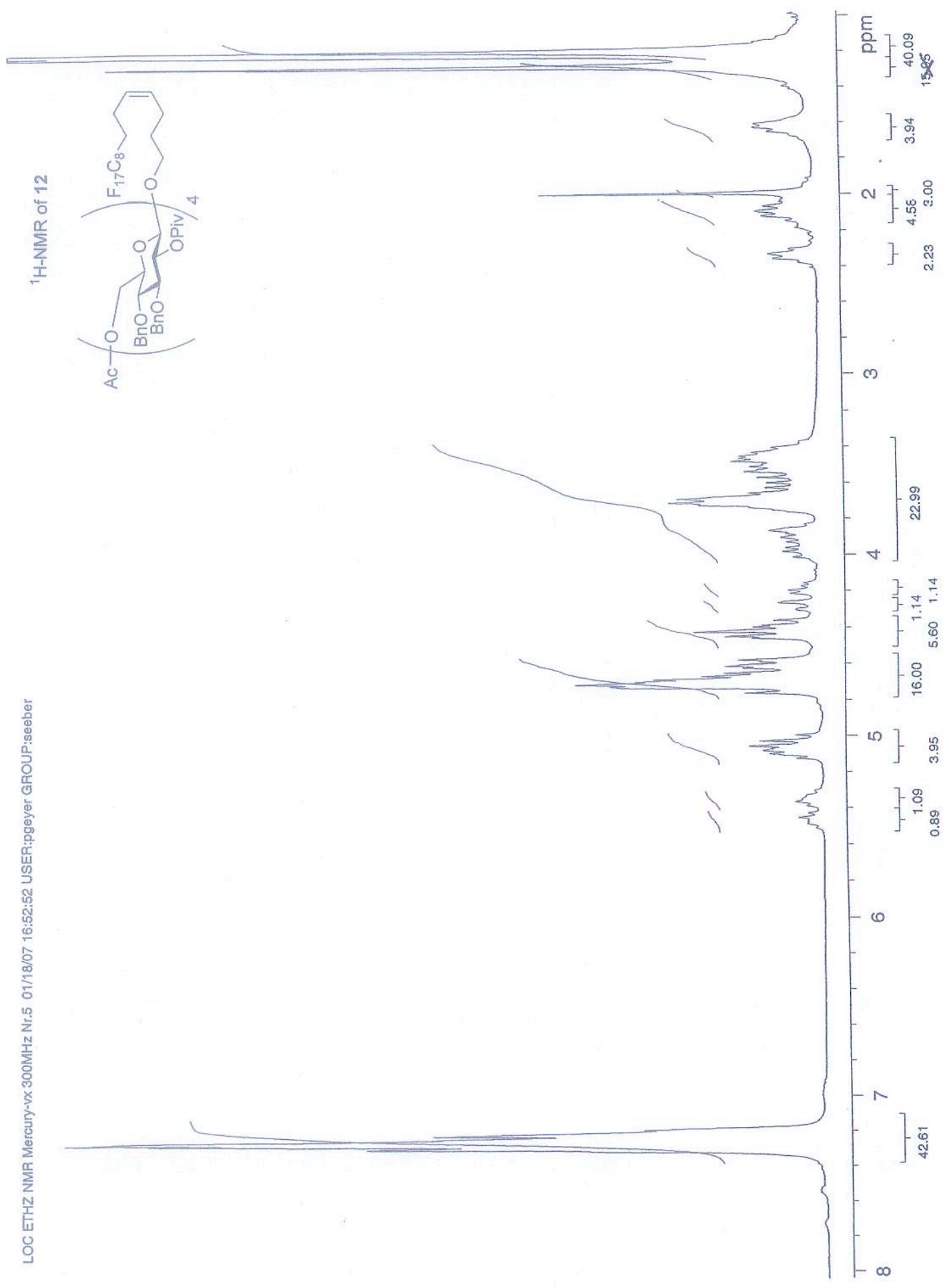



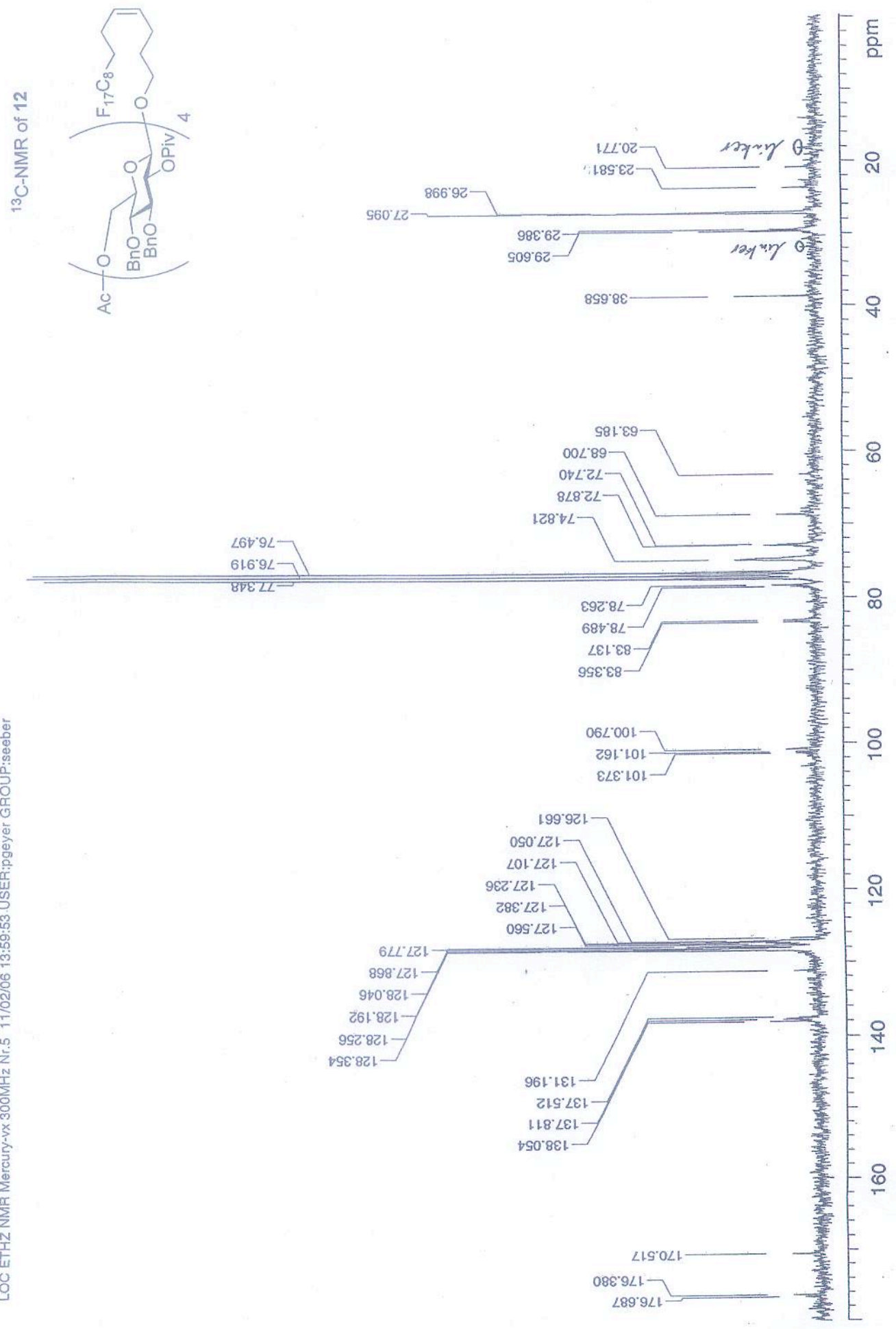
<smiles>[Y]=C1CCC1</smiles> 


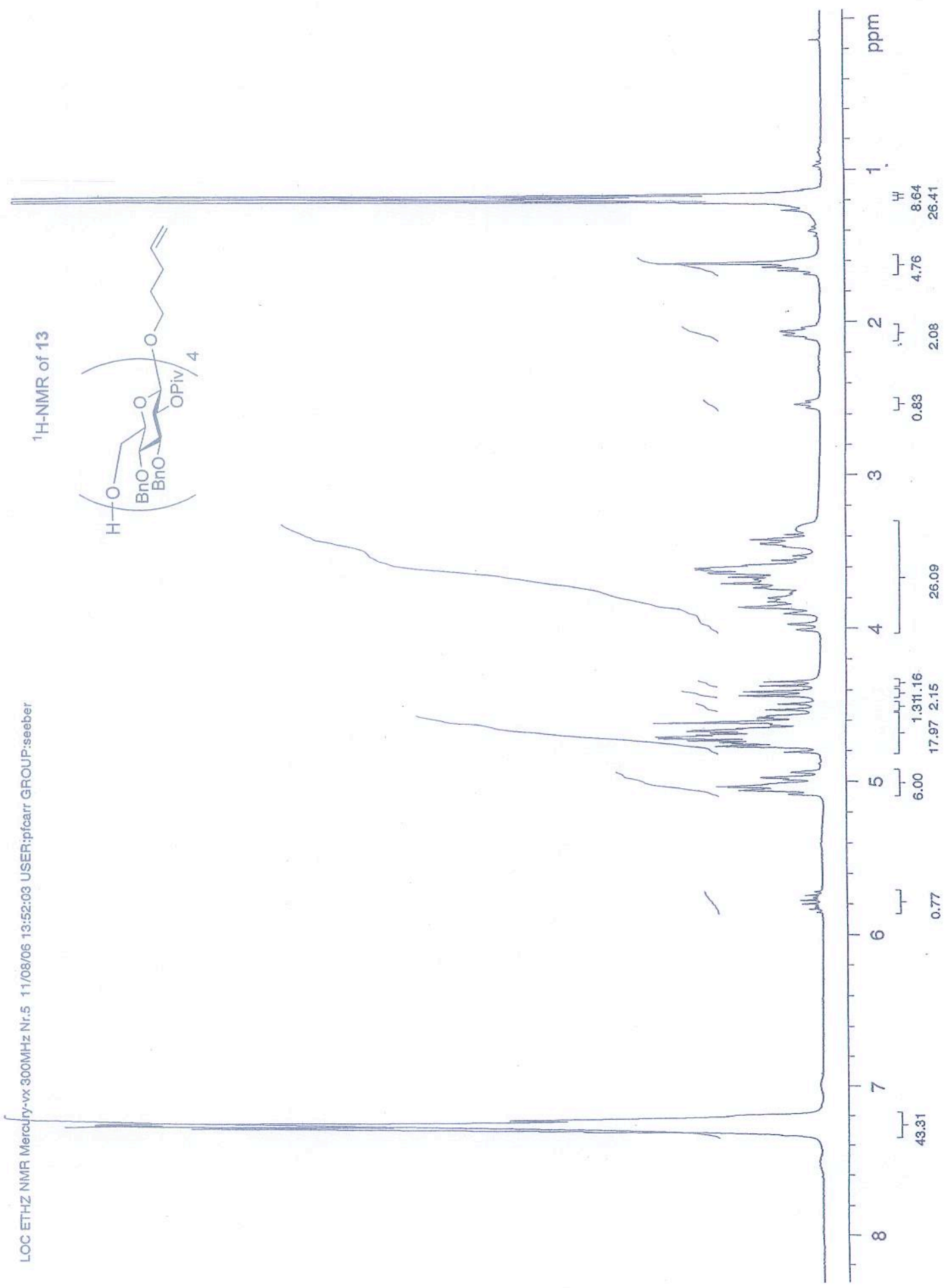




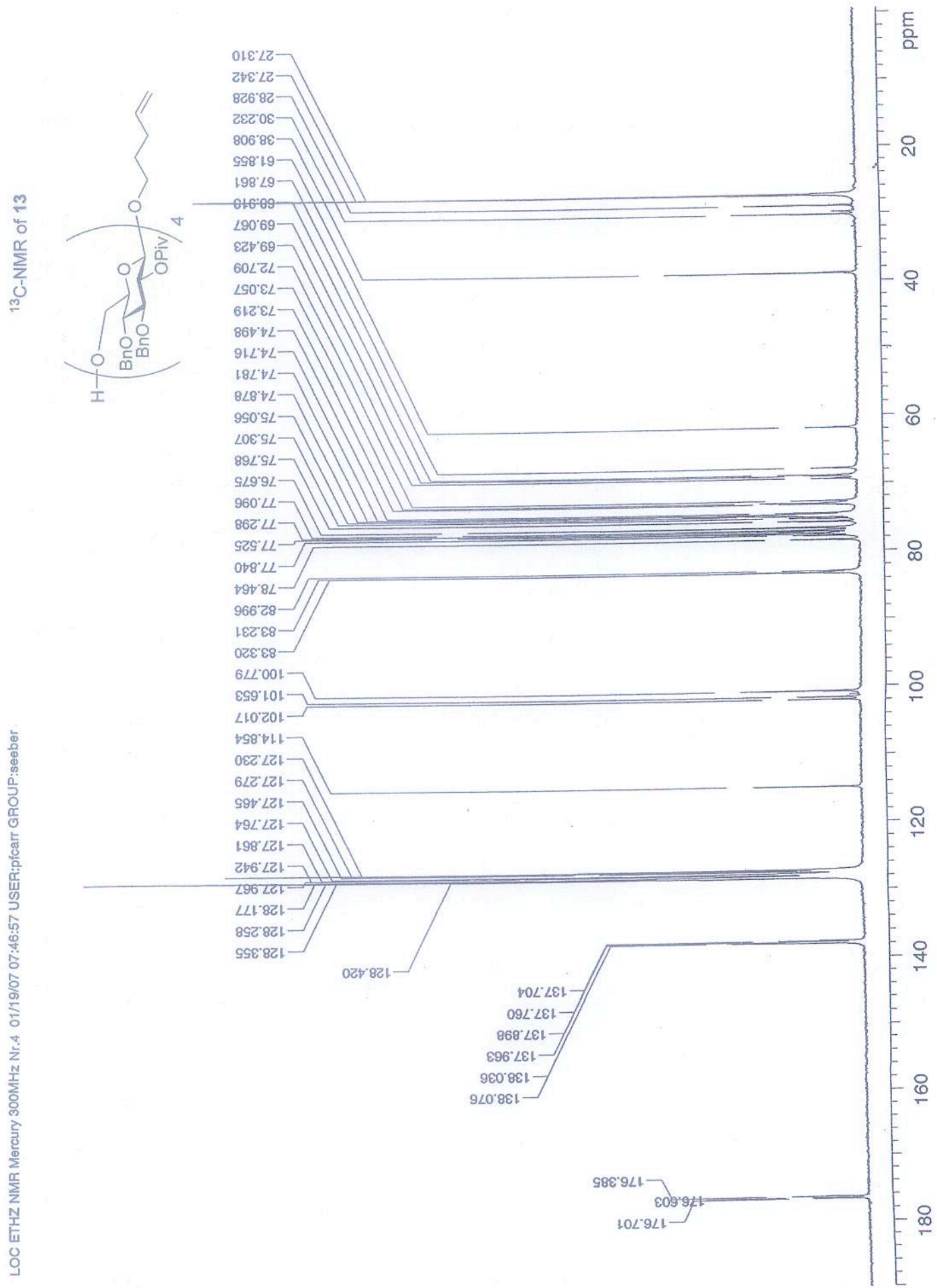




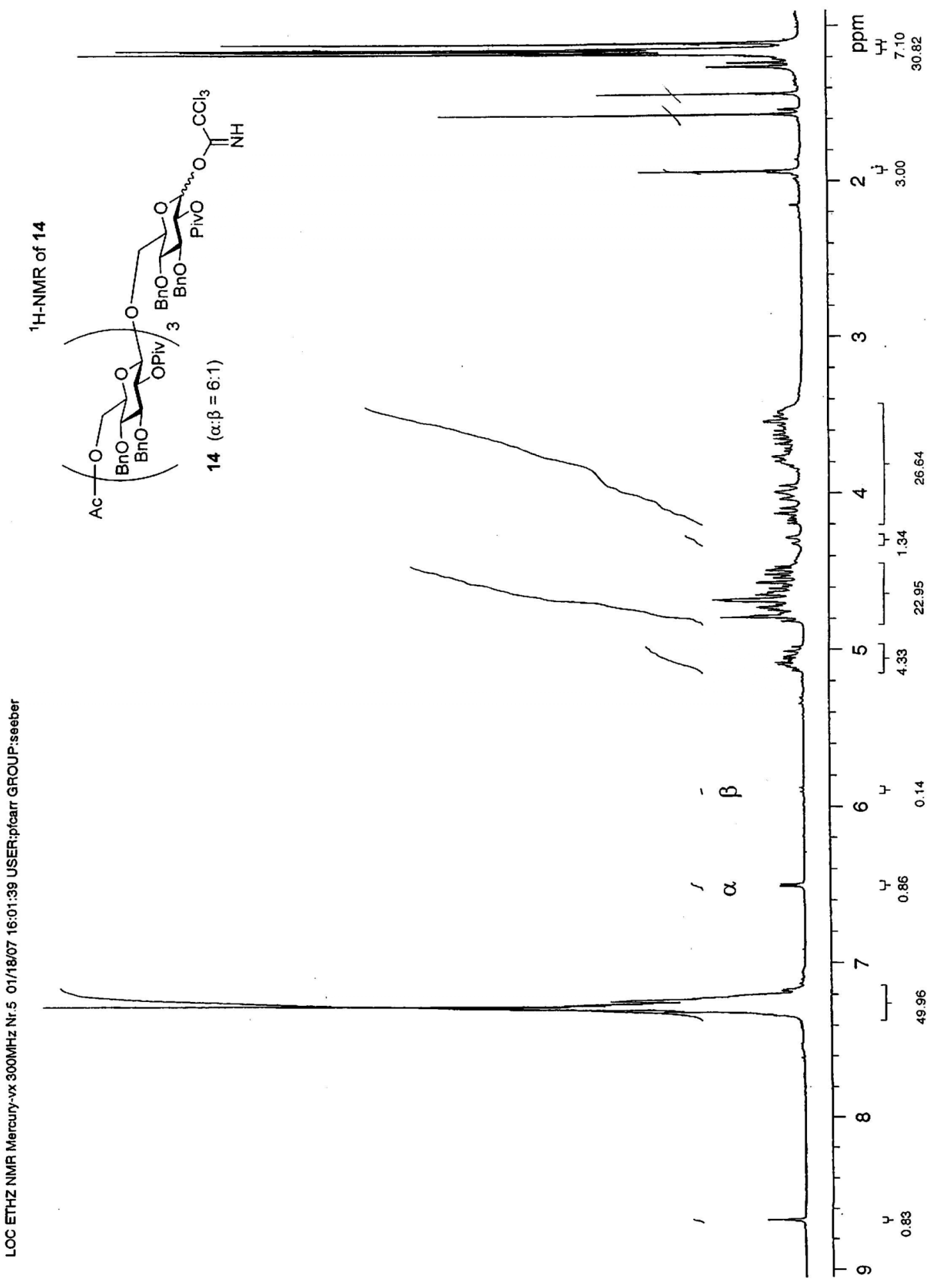




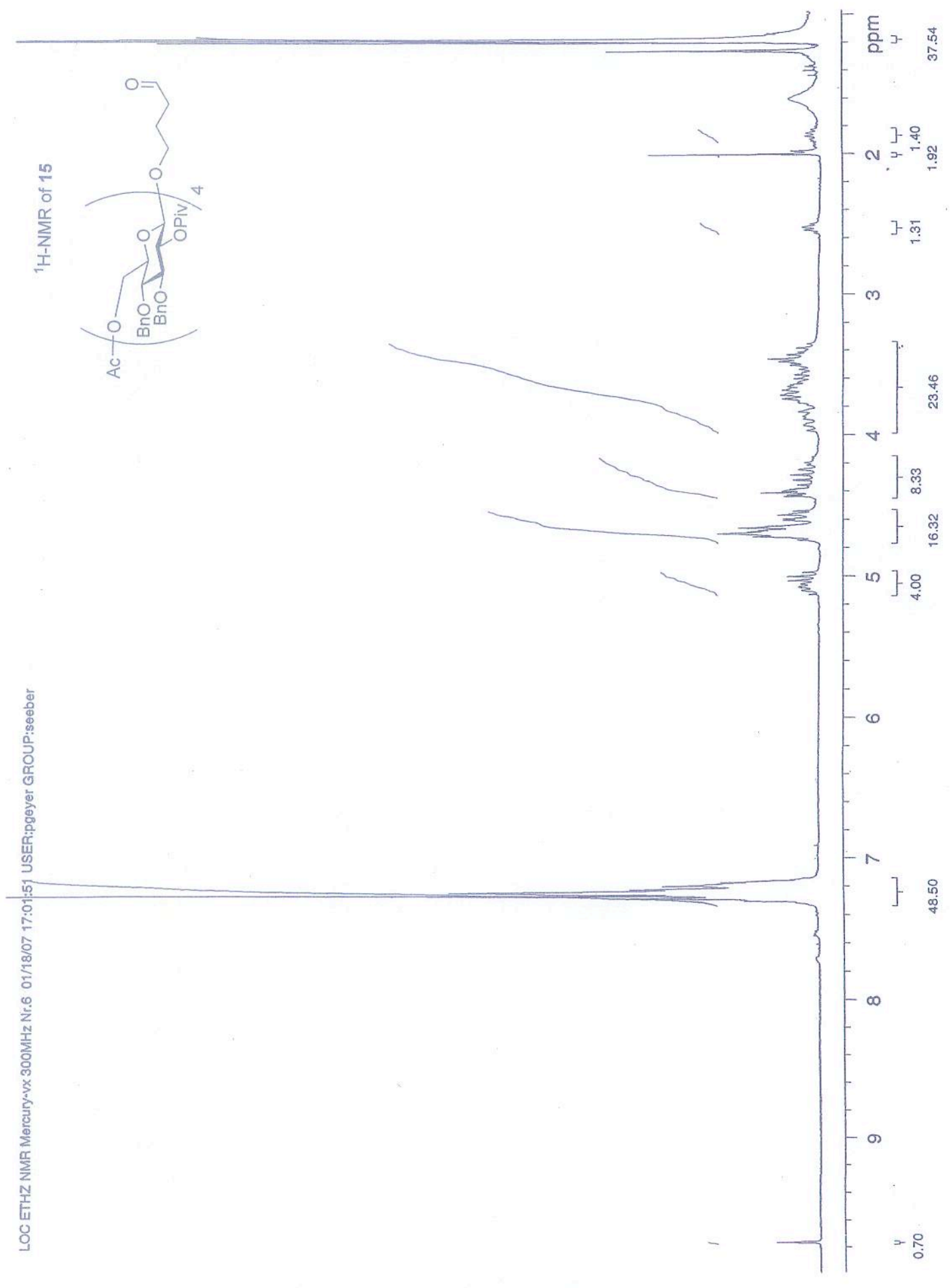




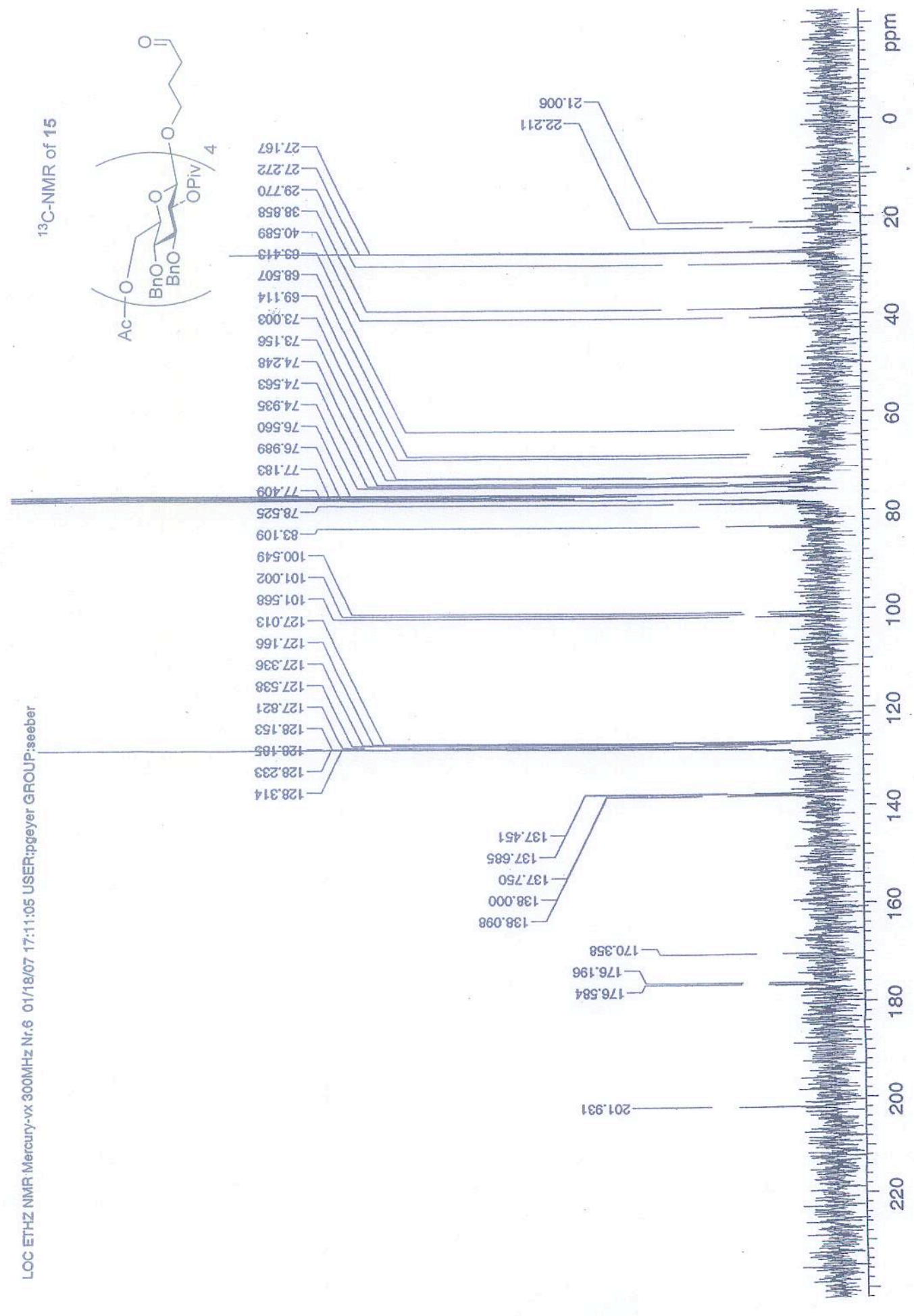

